Article

\title{
Impact of Citrate and Lipid-Functionalized Magnetic Nanoparticles in Dehydropeptide Supramolecular Magnetogels: Properties, Design and Drug Release
}

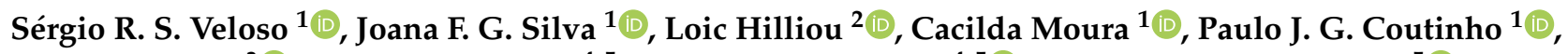 \\ José A. Martins ${ }^{3}{ }^{D}$, Martín Testa-Anta ${ }^{4,5}$, Verónica Salgueiriño ${ }^{4,5}{ }^{\mathbb{D}}$, Miguel A. Correa-Duarte ${ }^{5} \mathbb{D}$, \\ Paula M. T. Ferreira ${ }^{3}(\mathbb{D})$ and Elisabete M. S. Castanheira $1, * \mathbb{D}$
}

1 Centro de Física (CFUM), University of Minho, Campus de Gualtar, 4710-057 Braga, Portugal; sergioveloso96@gmail.com (S.R.S.V.); joanasilva.fg@gmail.com (J.F.G.S.); cmoura@fisica.uminho.pt (C.M.); pcoutinho@fisica.uminho.pt (P.J.G.C.)

2 Institute for Polymers and Composites, Department of Polymer Engineering, University of Minho, Campus de Azurém, 4800-058 Guimarães, Portugal; loic@dep.uminho.pt

3 Centro de Química (CQUM), University of Minho, Campus de Gualtar, 4710-057 Braga, Portugal; jmartins@quimica.uminho.pt (J.A.M.); pmf@quimica.uminho.pt (P.M.T.F.)

4 Departamento de Física Aplicada, Universidade de Vigo, 36310 Vigo, Spain; mtesta@uvigo.es (M.T.-A.); vsalgue@uvigo.es (V.S.)

5 CINBIO, Universidade de Vigo, 36310 Vigo, Spain; macorrea@uvigo.es

* Correspondence: ecoutinho@fisica.uminho.pt; Tel.: +351-253-604321

Citation: Veloso, S.R.S.; Silva, J.F.G.; Hilliou, L.; Moura, C.; Coutinho, P.J.G.; Martins, J.A.; Testa-Anta, M.; Salgueiriño, V.; Correa-Duarte, M.A.; Ferreira, P.M.T.; et al. Impact of Citrate and Lipid-Functionalized Magnetic Nanoparticles in Dehydropeptide Supramolecular Magnetogels: Properties, Design and Drug Release. Nanomaterials 2021, 11, 16. https://dx.doi.org/10.3390/nano 11010016

Received: 29 November 2020 Accepted: 21 December 2020 Published: 23 December 2020

Publisher's Note: MDPI stays neutral with regard to jurisdictional claims in published maps and institutional affiliations.

Copyright: () 2020 by the authors. Licensee MDPI, Basel, Switzerland. This article is an open access article distributed under the terms and conditions of the Creative Commons Attribution (CC BY) license (https: / / creativecommons.org/ licenses/by/4.0/).

\begin{abstract}
Currently, the nanoparticle functionalization effect on supramolecular peptide-based hydrogels remains undescribed, but is expected to affect the hydrogels' self-assembly and final magnetic gel properties. Herein, two different functionalized nanoparticles: citrate-stabilized $(14.4 \pm 2.6 \mathrm{~nm})$ and lipid-coated $(8.9 \pm 2.1 \mathrm{~nm})$ magnetic nanoparticles, were used for the formation of dehydropeptidebased supramolecular magnetogels consisting of the ultra-short hydrogelator Cbz-L-Met-Z- $\Delta$ Phe$\mathrm{OH}$, with an assessment of their effect over gel properties. The lipid-coated nanoparticles were distributed along the hydrogel fibers, while citrate-stabilized nanoparticles were aggregated upon gelation, which resulted into a heating efficiency improvement and decrease, respectively. Further, the lipid-coated nanoparticles did not affect drug encapsulation and displayed improved drug release reproducibility compared to citrate-stabilized nanoparticles, despite the latter attaining a stronger AMF-trigger. This report points out that adsorption of nanoparticles to hydrogel fibers, which display domains that improve or do not affect drug encapsulation, can be explored as a means to optimize the development of supramolecular magnetogels to advance theranostic applications.
\end{abstract}

Keywords: magnetic gels; drug release; magnetic hyperthermia; magnetic lipogels; supramolecular hydrogels; magnetic nanoparticles; self-assembly; nanoparticle functionalization

\section{Introduction}

Supramolecular magnetogels basically comprise two main components: the hydrogel and the magnetic nanoparticles. Following a stimulus, the self-assembly of the supramolecular hydrogelators is driven towards a kinetically-trapped intertwined fibrillar structure, such that solvent pocket microdomains are formed. This process takes place through the cooperative effect of different non-covalent intermolecular interactions: hydrogen bonding, van der Waals, electrostatic, and/or hydrophobic and aromatic interactions [1-7].

For example, dehydropeptide-based hydrogelators, such as the minimalist Cbz-LMet-Z- $\Delta$ Phe-OH (see Structure S1 in Supplementary Materials), have been used due to the simplicity of producing biocompatible systems at low cost, and its promising properties for drug delivery [8-10]. The aforementioned dehydropeptide uses of a non-polar amino acid, methionine, to induce hydrophobic collapse, while the dehydrophenylalanine moiety 
provides a means for $\pi-\pi$ interactions (like the $N$-capping group) and conformational constraints in the peptide backbone, meaning that it promotes the self-assembly into fibers and provides resistance to enzymatic degradation [8-10]. As assessed in this work, the hydrogel displays a moderate gelation kinetics and elastic modulus, which allows following the impact of the nanoparticles, so that diverse parameters can be optimized. Further, the gels can be formed in a skin $\mathrm{pH}$ range of 4-6 [11], thus being suitable for dermal application.

The retention or entrapment of nanoparticles during the gelation process allows for the tailoring and modulation of the matrix structure, along with the possibility of tuning different physical properties $[3,12,13]$. The modulation of the matrix structure also enables optimizing the loading of a wide variety of hydrophilic and hydrophobic drugs, reducing potential side effects, and enabling higher doses in therapy at the right location when using magnetogels for drug delivery. In this regard, taking advantage of the magnetic nanoparticles, the application of a magnetic field gradient allows the control and targeting of the nanosystem to a specific location [14-16], which can be further coupled with an alternating magnetic field (AMF), such that the nanoparticles can absorb energy and then release it as heat [17-20]. As a consequence, there is a synergistic effect that involves this magnetic hyperthermia and the subsequently associated enhancement of drug release [21-23], such that a much higher therapeutic efficiency can be attained. Along these lines, among transition metal ferrites, manganese ferrite nanoparticles exhibit very suitable magnetic properties, particularly in terms of the high magnetic susceptibility and in terms of magnetophoretic mobility, which render them as an appealing option to improve the supramolecular magnetogels for drug delivery applications [24,25]. Further, manganese ferrites display good biocompatibility and the large saturation magnetization reduces the required concentration of nanoparticles, thus averting side effects $[8,25]$.

Supramolecular magnetogels endorsed with bare nanoparticles were demonstrated to be easily prone to aggregation, which led us to limit the concentration of nanoparticles that were encapsulated [8,9]. Alternatively, the use of a thick shell coating the nanoparticles was confirmed to hamper the gelation process, with the additional cost of requiring a larger concentration of hydrogelator to compensate for the bulkier nanocomposite [10]. On the other hand, forcing the supramolecular design strategies to conjugate the nanoparticles with the hydrogelator molecules has also been considered [25-27]. In general, these existing strategies for supramolecular magnetogels either imply complex fabrication steps and/or fail on the homogeneous incorporation of high concentrations of magnetic material and target drugs, which strongly limit their usability in theranostics. Furthermore, the current literature on supramolecular magnetogels lacks exploration of the composite functionalization and its effect on supramolecular magnetic gels properties. Consequently, the large amounts of hydrogelator employed and the aggregation of nanoparticles in most of the reported systems have demonstrated lower heating efficiency, hindering the sought on-demand controlled release of the payload.

Doxorubicin is one of the most commonly used chemotherapeutic drugs in a wide variety of cancers. However, its various side effects (e.g., neutropenia and heart failures) lead to a worsening of the patient's quality of life, which can be improved through incorporation with drug delivery system to increase the safety profile of the therapy. Considering this need, a useful strategy could be the encapsulation of doxorubicin in liposomal systems, such as the liposomal formulation Doxil ${ }^{\circledR}$, or the use of citrate-stabilized nanoparticles, which strongly adsorb the positively charged drug molecules through electrostatic interactions [28].

Herein, supramolecular magnetogels endorsed with high nanoparticle concentration, employing a lipid coating (magnetoliposome-like structure) or citrate stabilization, are evaluated. Whereas the citrate stabilization provides electrostatic stabilization, the lipid coating assessment tackles the strategy, not only to ensure the steric stabilization of manganese ferrite nanoparticles, but also to provide the structure with enough hydrophobic cavities for an efficient drug loading and subsequent controlled drug diffusion. This option to ob- 
tain magnetogels systems, compared with the use of negatively-charged citrate-stabilized nanoparticles, demonstrates that the gelation becomes optimized, such that the concentration of stable magnetic nanoparticles within the gel is increased. As a consequence, besides improving the system stability and magnetic properties, the strategy demonstrates the role of the magnetic nanoparticles taking advantage of the hyperthermia they are responsible for, on drug release, using the antitumor drug doxorubicin.

\section{Materials and Methods}

\subsection{Synthesis Procedure of Magnetic Nanoparticles}

\subsubsection{Preparation of Citrate-Stabilized Manganese Ferrite Nanoparticles}

A modified synthesis of citrate-stabilized nanoparticles was adapted from reference [29]. Trisodium citrate dehydrate $(1 \mathrm{mmol})$ and $\mathrm{NaOH}(4 \mathrm{mmol})$ were added to $19 \mathrm{~mL}$ of ultrapure water at $100{ }^{\circ} \mathrm{C}$. A $1 \mathrm{~mL}$ aqueous solution of $\mathrm{FeSO}_{4} \cdot 7 \mathrm{H}_{2} \mathrm{O}(1.33 \mathrm{mmol})$ and $\mathrm{MnSO}_{4} \cdot \mathrm{H}_{2} \mathrm{O}$ (all reagents from Merck-Sigma, St. Louis, MO, USA) (0.66 mmol) was added, drop by drop, into the mixture under vigorous agitation. After $2 \mathrm{~h}$, the solution was cooled down to room temperature, washed through magnetic decantation with water/ethanol 1:1, and dried at $100^{\circ} \mathrm{C}$.

\subsubsection{Preparation of Lipid-Coated Manganese Ferrite Nanoparticles}

Lauric acid ( $1 \mathrm{mmol})$ and $\mathrm{NaOH}(4 \mathrm{mmol})$ were added to $19 \mathrm{~mL}$ of ultrapure water at $100{ }^{\circ} \mathrm{C}$. A $1 \mathrm{~mL}$ aqueous solution of $\mathrm{FeSO}_{4} \cdot 7 \mathrm{H}_{2} \mathrm{O}(1.33 \mathrm{mmol})$ and $\mathrm{MnSO}_{4} \cdot \mathrm{H}_{2} \mathrm{O}(0.66 \mathrm{mmol})$ was added, drop by drop, into the mixture under vigorous agitation. After $2 \mathrm{~h}$, the solution was cooled down to room temperature, washed through repeated centrifugation with water, and dried at $100{ }^{\circ} \mathrm{C}$. The stock solution was prepared by dispersion of the nanoparticles (4 mg) in $2 \mathrm{~mL}$ of $2 \mathrm{mM}$ L- $\alpha$-phosphatidylcholine (from egg yolk, egg-PC) (Merck-Sigma, St. Louis, MO, USA) solution through sonication at $190 \mathrm{~W}$. The lipid-coated nanoparticles were then washed and purified with ultrapure water by magnetic decantation.

\subsection{Self-Assembly of Magnetogels}

\subsubsection{Optimization of Hydrogel Gelation}

Gelation optimization was carried out through turbidity measurements at $500 \mathrm{~nm}$. The hydrogel and glucono- $\delta$-lactone (GdL) concentrations were screened. The self-assembly was induced by dissolving the hydrogelator in basic $\mathrm{pH}$ through the addition of $2 \mathrm{v} / \mathrm{v} \%$ $\mathrm{NaOH}(1 \mathrm{M})$ and, then, glucono- $\delta$-lactone $(\mathrm{GdL})$ was added to decrease the $\mathrm{pH}$ homogeneously. A solution of $0.05 \mathrm{wt} \%$ hydrogel was used to assess the fiber-catalyzed secondary nucleation. The aggregates fraction, $f(t)$, was defined as follows:

$$
f(t)=\frac{T_{\text {obs }}-T_{\text {free }}}{T_{\text {agg }}-T_{\text {free }}}
$$

where $T_{t}, T_{\text {free }}$ and $T_{\infty}$ stand for turbidity measured at $500 \mathrm{~nm}$ observed at time $t$, before the addition of GdL and when aggregated (turbidity at $t=5 \mathrm{~h}$ ), respectively. An empirical exponential decay function was fitted according to Equation (2) [30]:

$$
T(t)=\frac{T(\infty)}{\sqrt[v]{\left(1+v e^{-k_{e m p}\left(t-t_{m}\right)}\right)}}
$$

where $k_{\text {emp }}$ is the rate constant (inverse of the relaxation time) of fibril formation and $t_{m}$ is the point of the maximum elongation rate. This constant rate includes various steps and its interpretation between different systems is misleading. As a result, other models were fitted to understand the influence of the parameters on the nucleation and elongation rates. Saitô's fractional aggregation model has been successful in the aggregation studies of $\beta$-amyloid, calcitonin, prion, and $\alpha$-sinuclein [31-33]. At a concentration larger than 
critical micellar concentration $([M] \gg c m c)$, the aggregation can be described by a two-step mechanism scheme [33]:

$$
\begin{gathered}
\left.n_{0} M(\text { monomers }) \rightleftharpoons M_{n_{0}} \text { (micelle }\right) \stackrel{k_{n}}{\rightarrow} P_{n} \\
M+P_{n} \stackrel{k_{e}}{\rightarrow} P_{n+1} .
\end{gathered}
$$

In this mechanism, $k_{n}$ and $k_{e}$ represent the average nucleation and growth rate constants, $M$ is the monomer, $M_{n_{0}}$ represents the micelle (precatalytic form of the monomer), $P_{n}$ is the nucleus of fibril with $n$ monomer molecules, and $P_{n+1}$ is the extended fibril with $n+1$ monomer molecules; thus, $P$ is both a product and catalyst in the growth step reaction. The fibril formation can be described according to the equation:

$$
f(t)=\frac{\rho\left(e^{(1+\rho) k_{s} t}-1\right)}{1+\rho e^{(1+\rho) k_{s} t}}
$$

where $k_{s}=k_{e}[M]$ is the effective growth rate constant, $\rho=\frac{k_{n}}{k_{s}}$, and the initial condition is $f(0)=0$. The secondary nucleation was evaluated through the aggregation models of Knowles et al. [30,34] and Cohen et al. [35]. The former model describes the concentration of monomer in the fibers according to the equation:

$$
f(t)=1-e^{-C_{+} e^{k t}+C_{-} e^{-k t}+k_{n} m_{t o t}^{n_{c}-1} k_{-}^{-1}}
$$

where $m_{t o t}$ is the total hydrogelator concentration and $k=\sqrt{2 m_{t o t} k_{+} k_{-}}$. In the absence of fibrils, at the beginning of the aggregation,

$$
C_{ \pm}=\frac{ \pm k_{n} m_{t o t}^{n_{c}-1}}{2 k_{-}}
$$

where $k_{+}$is the polymerization rate and $k_{-}$is the secondary nucleation (fragmentation). The latter model describes the concentration of monomer in the fibers according to the equation:

$$
f(t)=1-\left(\frac{B_{+}+C_{+}}{B_{+}+C_{+} e^{\kappa t}} \frac{B_{-}+C_{+} e^{\kappa t}}{B_{-}+C_{+}}\right)^{\frac{k \infty}{\kappa k j \infty}}-e^{-k_{\infty} t}
$$

where $\kappa=\sqrt{2 k_{+} k_{2} m(0)^{n_{2}+1}}$ is associated with the secondary pathways, $k_{2}$ is the fibrilcatalyzed secondary nucleation, and $k_{2}=k_{-}$when $n_{2}=0, C_{ \pm}= \pm \lambda^{2} /\left(2 \kappa^{2}\right)$, $\lambda=\sqrt{2 k_{+} k_{n} m(0)^{n_{c}}}$ is related with the rate of formation of new aggregates through primary pathways, $B_{ \pm}=\left(k_{\infty} \pm k j_{\infty}\right) /(2 \kappa), k_{\infty}=\sqrt{2 \kappa^{2} /\left[n_{2}\left(n_{2}+1\right)\right]+2 \lambda^{2} / n_{c}}$, and $k_{\infty}=\sqrt{k_{\infty}^{2}-4 C_{+} C_{-} k^{2}}$. The parameters $n_{c}$ and $n_{2}$ describe the dependencies of the primary and secondary pathways, and $m(0)$ is the initial concentration of soluble monomers.

\subsubsection{Development of Magnetogels}

The prepared nanoparticles were added to the hydrogel solution at a final volume of $200 \mu \mathrm{L}$ and at the required concentration from a starting solution at $2 \mathrm{wt} \%$. All hydrogel/magnetogel solutions were left standing at room temperature until the gel phase was attained. Here, the unit $\mathrm{w} \mathrm{t} \%$ stands for $\mathrm{m} / \mathrm{v} \%$.

\subsection{Spectroscopic Measurements}

\subsubsection{General Methods}

Fluorescence measurements were carried out using a Horiba-Jobin Yvon Fluorolog 3 spectrofluorimeter (HORIBA Jobin Yvon IBH Ltd., Glasgow, UK), equipped with double excitation and emission monochromators, Glan-Thompson polarizers (HORIBA Jobin Yvon IBH Ltd., Glasgow, UK), and a temperature-controlled cuvette holder. Fluorescence 
emission spectra were corrected for the instrumental response of the system. The excitation of the hydrogelator was set at $280 \mathrm{~nm}$, and the emission spectrum was collected between $290 \mathrm{~nm}$ and $600 \mathrm{~nm}$ with a slit of $6 \mathrm{~nm}$ in both excitation and emission. Absorption spectra were recorded in a Shimadzu UV-3600 Plus UV-Vis-NIR spectrophotometer (Shimadzu Corporation, Kyoto, Japan).

The fluorescence quantum yield, $\Phi_{s}$, can be determined by Equation (7) (standard method) [36,37],

$$
\Phi_{s}=\frac{\left(A_{r} F_{s} n_{s}^{2}\right)}{\left(A_{s} F_{r} n_{r}^{2}\right)} \Phi_{r}
$$

where $A$ is the absorbance at the excitation wavelength, $F$ is the integrated emission area, and $n$ is the refraction index of the solvents. Subscripts $r$ and $s$ refer to the reference and sample compound, respectively. The absorbance value at excitation wavelength was always less than 0.1 in order to avoid inner filter effects. L-Tryptophan in aqueous buffer solution $(\mathrm{pH}=7.2)$ was used as a reference $\left(\Phi_{r}=0.14\right.$ at $\left.25^{\circ} \mathrm{C}\right)$ [38].

\subsubsection{Fluorescence Anisotropy Measurements}

The steady-state fluorescence anisotropy values, $r$, provide information on the average microviscosity of the gel matrix where the fluorophore is localized and can be determined by Equation (8) [39],

$$
r=\frac{I_{\mathrm{VV}}-G I_{\mathrm{VH}}}{I_{\mathrm{VV}}+2 G I_{\mathrm{VH}}}
$$

where $I_{\mathrm{VV}}$ and $I_{\mathrm{VH}}$ are the intensities of the emission spectra obtained with vertical and horizontal polarization, respectively (for vertically polarized excitation light), $I_{\mathrm{HV}}$ and $I_{\mathrm{HH}}$ are the emission intensities obtained with vertical and horizontal polarization (for horizontally polarized excitation light), and $G=I_{\mathrm{HV}} / I_{\mathrm{HH}}$ is the instrumental correction factor.

\subsubsection{FRET Measurements}

The drug incorporation into the magnetogels network was investigated by Förster Resonance Energy Transfer (FRET). FRET efficiency, $\Phi_{F R E T}$, defined as the proportion of donor molecules that have transferred their excess energy to acceptor molecules, can be expressed by Equation (9) [39],

$$
\Phi_{F R E T}=1-\frac{I_{D A}}{I_{D}}
$$

where $I_{D A}$ and $I_{D}$ are the donor integrated fluorescence intensities in the presence and absence of an acceptor, respectively. FRET efficiency can also be determined using the donor-acceptor intermolecular distance, $R_{D A}$, and the Förster radius (critical diameter), $R_{0}$, through Equation (10) [39],

$$
\Phi_{\text {FRET }}=\frac{1}{1+\left(\frac{R_{D A}}{R_{0}}\right)^{6}} .
$$

The Förster radius, $R_{0}$, the distance at which FRET efficiency is $50 \%$, can be determined by the spectral overlap, $J(\lambda)$ between the donor fluorescence emission and the acceptor absorption, according to Equations (11) and (12) (with $R_{0}$ in $\AA, \lambda$ in $n m, \varepsilon_{A}(\lambda)$ in $\mathrm{M}^{-1} \mathrm{~cm}^{-1}$ ) [39],

$$
\begin{gathered}
R_{0}=0.2108 \times\left[\kappa^{2} \Phi_{D} n^{-4} J(\lambda)\right]^{1 / 6} \\
J(\lambda)=\int_{0}^{\infty} I_{D}(\lambda) \varepsilon_{A}(\lambda) \lambda^{4} d \lambda
\end{gathered}
$$

where $\kappa^{2}=2 / 3$ is the orientational factor assuming random orientation of the dyes, $\Phi_{D}$ is the donor fluorescence quantum yield in the absence of energy transfer, $n$ is the refraction 
index of the medium, $I_{D}(\lambda)$ is the fluorescence spectrum of the donor normalized so that $\int_{0}^{\infty} I_{D}(\lambda) d \lambda=1$, and $\varepsilon_{A}(\lambda)$ is the molar absorption coefficient of the acceptor.

\subsection{Characterization Techniques}

\subsubsection{Scanning Transmission Electron Microscopy (STEM)}

STEM images were recorded using a NanoSEM-FEI Nova 200 (FEI Company, Hillsboro, OR, USA), operating at $15 \mathrm{kV}$, coupled to an Electron Dispersive Spectroscopic analyzer (EDS) and Electron Backscatter Diffraction EDAX-Pegasus X4M analyser (AMETEK Inc., Berwyn, PA, US) and detection system (EBSD) at SEMAT (Serviços de Caracterização de Materiais, Guimarães, Portugal). After preparation of the hydrogel, a small portion of each sample was placed onto a TEM 400 mesh copper grid with Formvar/Carbon (ref. S162-4 from Agar Scientific), held by tweezers and the excess solution was cleaned. The processing of STEM images was performed using ImageJ software (National Institutes of Health (NIH), version 1.52p, Bethesda, MD, USA), which consisted of enhancing local contrast and adjusting brightness followed by a manual selection of fibers.

\subsubsection{X-ray Diffraction}

A conventional PAN'alytical X'Pert PRO diffractometer (Malvern Panalytical Ltd., Malvern, UK) was used for X-ray diffraction (XRD) analyses, operating with $\mathrm{Cu} \mathrm{K}_{\alpha}$ radiation, in a Bragg-Brentano configuration.

\subsubsection{Raman Spectroscopic Measurements}

Raman spectroscopy was used to assess the effect of nanoparticles in the secondary structure of the hydrogel fibers. Measurements were performed at room temperature with a Jobin Yvon T64000 triple Raman Spectrometer (HORIBA Jobin Yvon IBH Ltd., Glasgow, UK), equipped with a liquid nitrogen cooled charge couple device (CCD) detector, with a resolution better than $1 \mathrm{~cm}^{-1}$. The excitation line, $514.5 \mathrm{~nm}$, of an argon ion laser was focused onto the sample using a $\times 50$ objective (focused to $\sim 1.5 \mu \mathrm{m}$ of diameter) of an Olympus Microscope BHSM (Olympus Corporation, Tokyo, Japan) in a backscattering geometry. The spectra were acquired with a measured power of about $350 \mu \mathrm{W}$ on the sample, with a spectral acquisition time of $45 \mathrm{~s}$ averaged over 10 scans, over the range $770-1800 \mathrm{~cm}^{-1}$.

\subsubsection{Magnetic Properties}

Magnetic measurements were performed using a SQUID magnetometer from Quantum Design (Quantum Design Inc., San Diego, CA, USA). The magnetization dependence with temperature in zero-field-cooling ( $\mathrm{ZFC}$ ) and field-cooling $(\mathrm{FC})$ conditions was performed at 100 Oe in the $10-320 \mathrm{~K}$ range. Hysteresis loops were measured at different temperatures up to an external field of $50 \mathrm{kOe}$.

\subsubsection{Rheology}

The viscoelastic characterization of gels was performed with a stress-controlled rotational rheometer Anton Paar MCR300 (Anton Paar GmbH, Graz, Austria). Liquid samples were loaded into the Couette geometry of the rheometer and temperature was kept at $25{ }^{\circ} \mathrm{C}$ during testing. After a five hour rest period ensuring gel setting and structural equilibrium of samples, a sweep in the strain amplitude was performed from $0.001 \%$ to $500 \%$ to assess the linear regime of viscoelasticity and the large amplitude oscillatory strain (LAOS) regime.

\subsection{Drug Release Assays}

\subsubsection{Incorporation of Doxorubicin}

To study the incorporation and microenvironment of doxorubicin in gels through fluorescence spectroscopy, the drug was added to gel solutions prior to gelation, for a final concentration of $20 \mu \mathrm{M}$ (to guarantee that fluorescence intensity is proportional to 
concentration). From the hydrogelator solution, $200 \mu \mathrm{L}$ were transferred to a fluorescence microcuvette and left standing until the gel was formed.

\subsubsection{Drug Release to $\mathrm{pH}=7$ Buffer}

To assess doxorubicin release through fluorescence spectroscopy, gels $(100 \mu \mathrm{L})$ loaded with $0.1 \mathrm{mM}$ doxorubicin were prepared and left stabilizing overnight in Amicon ${ }^{\circledR}$ Ultra$0.5 \mathrm{~mL}$ centrifugal filters (MilliporeSigma, St. Louis, MO, USA) with a $0.1 \mu \mathrm{m}$ pore size. Then, the filter tube was immersed in $\mathrm{pH}=7$ buffer $(800 \mu \mathrm{L})$ to keep $\mathrm{pH}$ constant (besides neutralizing the gels), and left standing at room temperature, with or without an alternating magnetic field (AMF). The AMF was generated in a custom-designed solenoid device (800 turns per meter, length: $31 \mathrm{~cm}$ and internal diameter: $4.8 \mathrm{~cm}$ ) by applying an alternating electric current. A magnetic field of $2.98 \mathrm{mT}$ at $1000 \mathrm{kHz}$ was used. Aliquots were taken and replaced with $\mathrm{pH}=7$ buffer, then fluorescence was measured to determine the concentration at each time point. Release profile assays were performed in triplicate.

\section{Results}

\subsection{Optimization of Hydrogel Gelation Kinetics}

Turbidity kinetic assays were carried out to optimize hydrogel gelation, which is required to ensure quasi-homogeneous encapsulation of nanocomposites. Initially, the hydrogelator molecules were majorly organized in a mixture of micelles/aggregates and free monomer as suggested by fluorescence emissions at $450 \mathrm{~nm}$ and $360 \mathrm{~nm}$, respectively (Figure 1A).
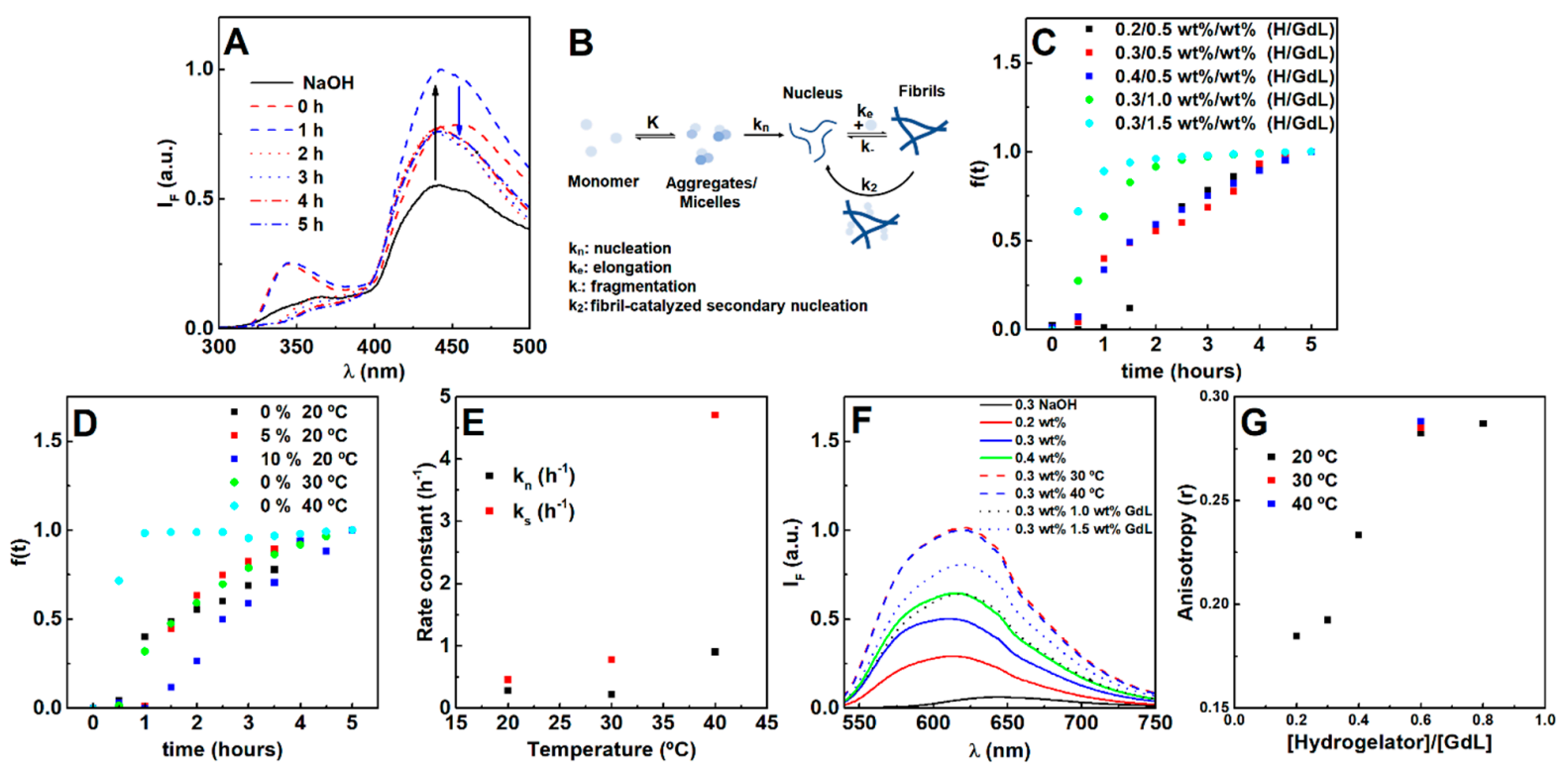

Figure 1. (A) Fluorescence emission spectra of the hydrogelator $(0.3 \mathrm{wt} \%)$ over time after the gelation trigger $(0.5 \mathrm{wt} \%)$. (B) Scheme of the evaluated pathways during the gelation process, where the fibril-catalyzed secondary nucleation contribution was found to be negligible. (C) Gelation kinetic profile dependence on hydrogelator and GdL concentration, (D) fibril concentration and temperature. (E) Average nucleation and elongation rate constants obtained from the Saitô's aggregation model fitting to turbidity profiles at increasing temperatures (hydrogel $0.3 \mathrm{wt} \%$, GdL $0.5 \mathrm{wt} \%$ ). Fluorescence emission $(\mathbf{F})$ and anisotropy $r(\mathrm{G})$ of Nile Red $(2 \mu \mathrm{M})$ in hydrogels prepared at different hydrogelator-to-GdL ratios and temperatures (fixed hydrogel and GdL concentration).

Once gelation is initiated (after the addition of GdL), both emission bands increase, indicating the occurrence of a reorganization process. Here, the models of Knowles et al. [34] and Cohen et al. [35] (commonly used for $\beta$-amyloid aggregation) were also used as a strategy to assess the possible occurrence and influence of monomer independent ( $\mathrm{k}_{-}$; 
fragmentation) and/or monomer dependent ( $\mathrm{k}_{2} ;$ fibril-catalyzed secondary nucleation) secondary pathways (Figure 1B).

The gelation is characterized by a sigmoidal profile, which is a common feature of fibrillation processes comprising a nucleation and elongation phase (Figure 1C,D) [30]. Increasing the hydrogelator concentration at the same $\mathrm{GdL}(0.5 \mathrm{wt} \%)$ concentration resulted in an increase of the nucleation rate, while the elongation rate decreased. The increase in GdL concentration promoted both the nucleation and elongation phases. Hereby, the primary nucleation process is dependent on both the hydrogelator and GdL, while the elongation process is majorly affected by the GdL. The elongation phase dependence on GdL concentration (for a fixed hydrogelator concentration) demonstrates that its rate can be increased if more protons are made available over time, considering that GdL proton dissociation is also a kinetically-dependent process [40]. Such results evidence that an increase of hydrogelator concentration has to be accompanied by an increase of GdL concentration to keep the molar equivalents, thus favoring both nucleation and elongation. Notably, by increasing both hydrogelator and GdL concentrations, no major differences were obtained for the final $\mathrm{pH}$, while increasing GdL alone strongly decreased $\mathrm{pH}$ (Table S1 in Supplementary Materials).

A weak scaling of the half-time with the initial monomer concentration was obtained $(-0.63)$, which is characteristic of monomer independent secondary pathways (such as fragmentation). Yet, the light scattering kinetic profiles cannot be satisfactorily matched using fixed $k_{n} / k_{-}$and $k_{+} k_{n}$ parameters in the Knowles' aggregation model (Figure S1 in Supplementary Materials) [35]. The addition of pre-formed fibrils inhibited the average nucleation phase and enhanced the average elongation phase (Figure 1D), i.e., the secondary nucleation rate can be neglected. Further, increasing the hydrogelator decreased both secondary pathways rates, while GdL enhanced the monomer independent pathway and inhibited the monomer-dependent pathway (Table S2 in Supplementary Materials). Nonetheless, selfassembly can be majorly attributed to the primary pathways. Temperature exponentially affected the average nucleation and elongation phase, which is associated with a faster GdL proton dissociation (Figure 1E) [40]. As a result, overall gelation can be enhanced by increasing both GdL and hydrogelator concentrations and preparing the gel at 30 or $40{ }^{\circ} \mathrm{C}$, which favors the primary pathways (nucleation and elongation).

The dye Nile Red was used to evaluate the effect of preparation conditions on the microenvironment, as it is a solvatochromic probe that has almost negligible emission in water, but intensely emits fluorescence in non-polar environments [41-44], as observed after hydrogel formation (Figure 1F). Furthermore, the emission is accompanied by a blue-shift with a reduction of polarity [41-44]. Here, Nile Red is localized in a microenvironment with a polarity between acetone and ethanol [41]. The higher Nile Red fluorescence emission intensity on the gels prepared at $30^{\circ} \mathrm{C}$ and $40^{\circ} \mathrm{C}$ suggests that more hydrophobic regions were made available. The fluorescence anisotropy values reveal similar fluidity compared to the hydrogel prepared at room temperature. Although increasing GdL (fixed hydrogelator concentration at $0.3 \mathrm{wt} \%$ ) also contributed for more hydrophobic regions, the microfluidity was lower than that obtained by increasing the hydrogelator concentration (fixed GdL concentration at $0.5 \mathrm{wt} \%$ ). As such, increasing both GdL and hydrogelator concentrations ( 0.4 hydrogelator-to-GdL ratio or higher) promotes more hydrophobic regions with higher microviscosity.

\subsection{Nanoparticles Characterization}

Nanoparticles of manganese ferrite with different coatings were prepared using different synthetic methods, and are named, from now on, as citrate-stabilized or lipid-coated manganese ferrite nanoparticles. The X-ray diffraction (XRD) patterns of both samples present well-defined peaks (Figure 2A,B) characteristic of a crystalline structure, which was obtained without calcination. The diffraction peaks of the $\mathrm{MnFe}_{2} \mathrm{O}_{4}$ crystalline spinel structure are observed at $2 \theta=29.7^{\circ}(220), 34.9^{\circ}(311), 36.5^{\circ}(222), 42.5^{\circ}(400), 52.7^{\circ}$

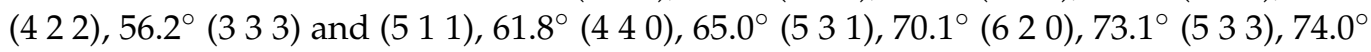


(6 2 2), $78^{\circ}$ (4 4 4), 85.6 (6 4 2), 88.5 (7 31 ) and (5 5 3), according to CIF file 2300618 (space group Fd-3m). Rietveld analysis was performed using the FullProf software suite, confirming the spinel structure. As in previous works $[45,46]$, we considered it to be important to use microabsorption correction [47], resulting in fits with good $R_{F}$ values. The calculated parameters are presented in Table S3 in Supplementary Materials, offering a larger size of the crystalline domains in the nanoparticles that are citrate-stabilized. Additional diffraction peaks were observed for the lipid-coated nanoparticles, occurring at positions similar to those reported for layered manganese laurate [48], which can be ascribed to ordered lauric acid molecules at the surface of manganese ferrite.

The UV-visible absorption spectra of the prepared nanoparticles are represented in Figure 2C. From the Tauc plot (inset of Figure 2C), the optical band gap $\left(E_{g}\right)$ between the citrate-stabilized and lipid-coated manganese ferrite nanoparticles was determined and a linear relation was obtained for an indirect semiconductor with a band gap of $1.13 \mathrm{eV}$ and $1.19 \mathrm{eV}$, respectively, which are similar to the previous reported value of $1.08 \mathrm{eV}$ [49].

The sedimentation profiles for bare, lipid-coated, and citrate-stabilized nanoparticles are displayed in Figure 2D. The dependence of the sedimentation rate on nanoparticle concentration (obtained through fitting of a Becquerel function or compressed hyperbola) [50] is included (values are reported in Table S4 in Supplementary Materials). The citrate-stabilized nanoparticles sedimentation profile suggests the occurrence of nanoparticles aggregation into stable agglomerates [51,52], which settle down at a faster rate than single nanoparticles. The lipid-coated nanoparticles show a sedimentation rate independent of the used concentration range $(0.025-0.2 \mathrm{wt} \%)$. Hereby, the longer-term stability of the lipid-coated nanoparticles is expected to provide homogeneous gels at higher concentration of nanoparticles, compared to the ones that are citrate-stabilized.
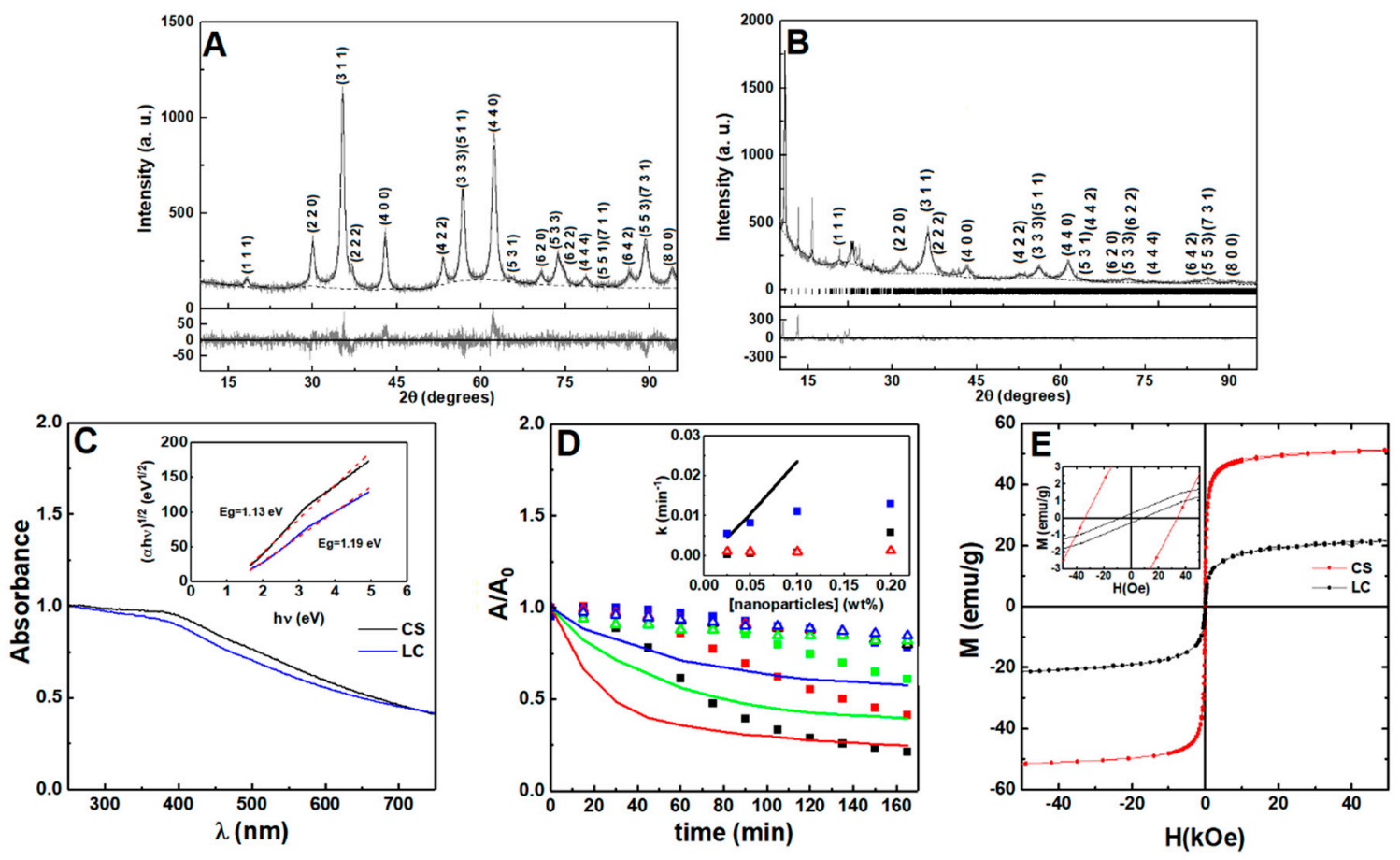

Figure 2. X-ray diffraction pattern of (A) citrate-stabilized and (B) lipid-coated manganese ferrite nanoparticles. Gray lines: experimental patterns; black lines: fitted patterns; dotted lines: fitted background; the vertical lines in (B) are the Bragg diffraction positions of manganese laurate. (C) Absorption spectra of citrate-stabilized (CS, black line) and lipid-coated (LC, blue line) $\mathrm{MnFe}_{2} \mathrm{O}_{4}$ nanoparticles. Inset: Tauc plot of citrate-stabilized and lipid-coated nanoparticles. (D) Sedimentation profiles of the citrate-stabilized (squares), lipid-coated (triangles), and bare (line) $\mathrm{MnFe}_{2} \mathrm{O}_{4}$ nanoparticles at $0.2 \mathrm{wt} \%$ (black), $0.1 \mathrm{wt} \%$ (red), $0.05 \mathrm{wt} \%$ (green), and $0.025 \mathrm{wt} \%$ (blue). Inset: sedimentation rate dependence on nanoparticle concentration. The citrate-stabilized aggregation rate is included (black squares). (E) Magnetization hysteresis loops of citrate-stabilized and lipid-coated manganese ferrite nanoparticles measured at room temperature $(\mathrm{T}=300 \mathrm{~K})$. Inset: Enlargement of the loops in the low field region. 
The magnetic hysteresis loops show that the saturation magnetization $(\mathrm{emu} / \mathrm{g})$ is higher for the citrate-stabilized nanoparticles than for the lipid-coated nanoparticles (Figure 2E), which can be explained by taking a different stoichiometry into account in the manganese ferrite in the two samples, as pointed out by the different lattice constant obtained in the X-ray diffraction analysis. A smaller size and a higher $w \mathrm{t} \%$ of organic matter present in the second sample can also influence the final value of saturation magnetization. Consequently, considering that $S A R \propto \mathrm{M}_{\mathrm{s}}{ }^{2}$ (SAR-specific absorption rate), the lipid-coated nanoparticles are expected to have lower heating efficiency than the citrate-stabilized nanoparticles $[53,54]$. The low $\mathrm{M}_{\mathrm{r}} / \mathrm{M}_{\mathrm{S}}$ ratio, of around 0.1 (see Table S5 in Supplementary Materials), is an indication that both types of nanoparticles display a superparamagnetic behavior [54].

\subsection{Development of Magnetogels}

An empirical equation was used to assist the estimation of the conditions required to maximize the homogeneity of the gel (see deduction, discussion and Figure S2 in Supplementary Materials). Figure 3 displays plots of the obtained $k_{e m p} / k_{\text {sed }}$ for various nanoparticle concentration and GdL-to-hydrogelator concentration ratio. The estimation implies that the gelation conditions have to guarantee a $\mathrm{k}_{\mathrm{emp}} / \mathrm{k}_{\mathrm{sed}}>41.7$, so that when gel fraction $f(t)$ attains 0.9 , the nanoparticles suspended fraction is also at 0.9 (if $v=1$ and $a=0.5$ is assumed).
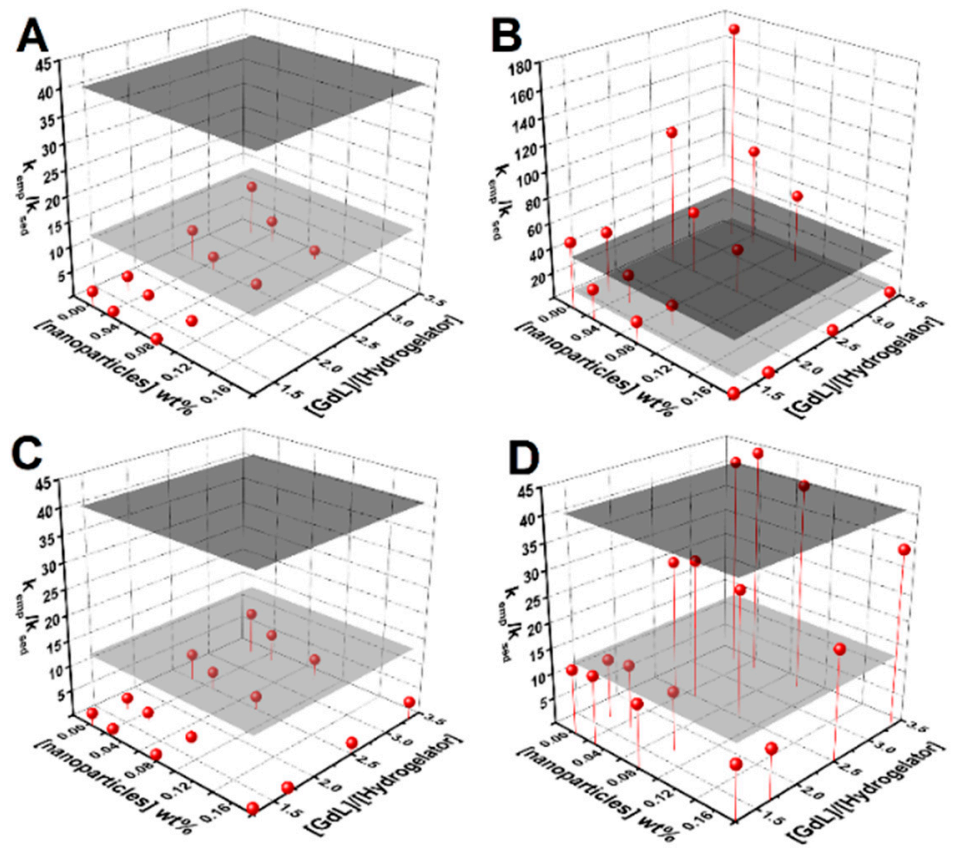

Figure 3. Surface plots of $k_{\mathrm{emp}} / \mathrm{k}_{\text {sed }}$ dependence on nanoparticle concentration and the GdL-tohydrogelator concentration ratio for: (A) bare nanoparticles, (B) the citrate-stabilized nanoparticles aggregation rate, $(\mathbf{C})$ the sedimentation rate, and (D) lipid-coated nanoparticles. The gray planes define the estimated $\mathrm{k}_{\mathrm{emp}} / \mathrm{k}_{\text {sed }}$ required to guarantee that when the gel is about $10 \%$ of gelation completion, $90 \%$ of the nanoparticles remains in suspension $(v=1, a=0.5$, dark gray plane; $v=0.5$, $\mathrm{a}=0.5$, gray plane).

Gels prepared with $1.5 \mathrm{wt} \% \mathrm{GdL}$ and $0.3 \mathrm{wt} \%$ hydrogelator retained the nanoparticles, though the $\mathrm{pH}$ value was lower than 4 . Increasing the hydrogelator content to $0.5 \mathrm{wt} \%$ and reducing GdL to $1 \mathrm{wt} \%$ (has higher microviscosity) allowed the preparation of homogeneous magnetogels at $0.1 \mathrm{wt} \%$ of nanoparticles, with a $\mathrm{pH}$ of $\sim 5$. The $0.2 \mathrm{wt} \%$ content of nanoparticles can also be prepared at $0.5 \mathrm{wt} \%$ of hydrogelator and $1 \mathrm{wt} \%$ of GdL but preparation at $30^{\circ} \mathrm{C}$ or $40^{\circ} \mathrm{C}$ is required (yields $\mathrm{k}_{\mathrm{emp}} / \mathrm{k}_{\text {sed }}$ larger than 200 ). Nonetheless, 
the parameter $v$ obtained from curve fitting was around 0.5 , thus decreasing the required $\mathrm{k}_{\mathrm{emp}} / \mathrm{k}_{\text {sed }}$ to 14 , which allowed the possibility of obtaining quasi-homogeneous gels at 0.1 $\mathrm{wt} \%$ of nanoparticles at a [GdL]/[hydrogelator] ratio of 2 (see magnetogels and respective $\mathrm{pH}$ values in Figure S3 in Supplementary Materials).

\subsection{Gels Microviscosity}

The effect of the nanoparticles in the gels matrix microenvironment was studied using Nile Red as a fluorescence probe, while considering its sensitivity to polarity and viscosity $[55,56]$. In the $20-40{ }^{\circ} \mathrm{C}$ temperature range, no major fluorescence emission decay changes were observed, which might be associated with the structure maintaining its integrity. A steep fluorescence emission decrease indicated that the phase transition occurs above $45^{\circ} \mathrm{C}$ for the hydrogel and the citrate-stabilized nanoparticle-containing magnetogels, while in the lipid-coated nanoparticle-containing magnetogels, it was shifted to $50{ }^{\circ} \mathrm{C}$ (Figure 4A). The fluorescence anisotropy increases at the phase transition temperature, which might be associated with a fluorescence emission lifetime decrease (Figure 4B) [57].
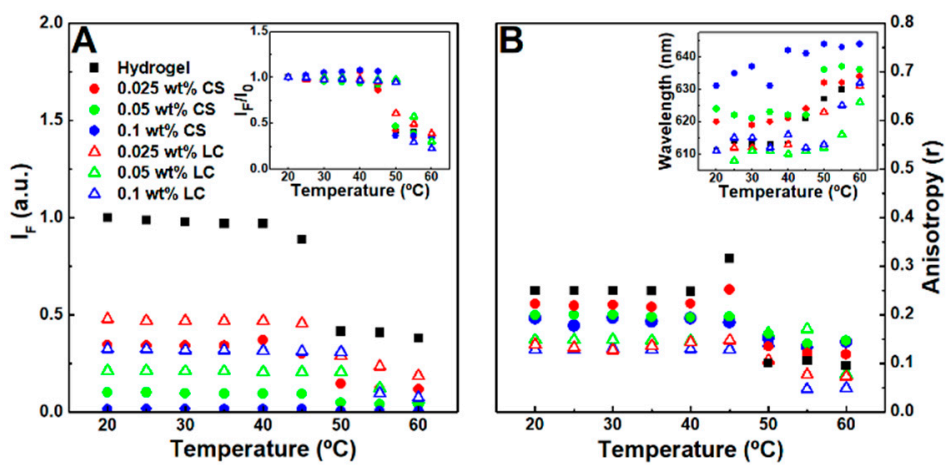

Figure 4. (A) Nile Red $(2 \mu \mathrm{M})$ maximum fluorescence emission dependence on the temperature of hydrogel, citrate-stabilized (CS), and lipid-coated (LC) nanoparticles containing magnetogels. Inset: Normalized maximum fluorescence emission dependence on the temperature of the respective gels. (B) Nile Red fluorescence anisotropy dependence on temperature. Inset: Nile Red maximum emission wavelength dependence on temperature.

The results suggest that citrate-stabilized nanoparticles destabilized the hydrophobic domains, leading to a microviscosity reduction and polarity increase of the cavities where Nile Red is localized, which might occur through hydrogen bonding and ionic interaction between the nanoparticles and the fibers. The polarity in the hydrogel is similar to acetone, which was changed towards ethanol after addition of citrate-stabilized nanoparticles. Furthermore, after phase transition and at $0.1 \mathrm{wt} \%$ of citrate-stabilized nanoparticles, the Nile Red emission wavelength $(640 \mathrm{~nm})$ became close to the reported maximum wavelength in water $(657 \mathrm{~nm})$ [42]. The lipid-coated nanoparticles induced a lower fluorescence anisotropy than citrate-stabilized nanoparticles and one that is similar to the reported anisotropy values of Nile Red in mixed vesicles and micelle membranes [44], thus suggesting that lipid-fiber domains are formed with a polarity similar to the fibers and a viscosity near that of membranes.

\subsection{Gels Secondary Structure}

The Raman spectra of the hydrogels and magnetogels ( $0.1 \mathrm{wt} \%$ of nanoparticles) were obtained to assess influence of nanoparticles in the secondary structure, which are displayed in Figure 5. Reported Raman shifts of the phenylalanine phenyl ring and methionine side chain $\left(\mathrm{CH}_{3}\right.$ deformation at $1440 \mathrm{~cm}^{-1}$ and $\mathrm{CH}_{2}$ wagging at $\left.1320 \mathrm{~cm}^{-1}\right)$ are also displayed $[58,59]$. Structural changes upon the addition of lipid-coated nanoparticles were suggested by the appearance of a band at $982 \mathrm{~cm}^{-1}$. Gaussian curves were fitted to the major phenyl ring signal at around $1003 \mathrm{~cm}^{-1}$ (see Figure S4 in Supplementary 
Materials). A blue shift and decreasing cross-section were observed with an increasing nanoparticle concentration. The latter effect can be associated with an increasing exposure of the aromatic rings to a more hydrated environment [60].

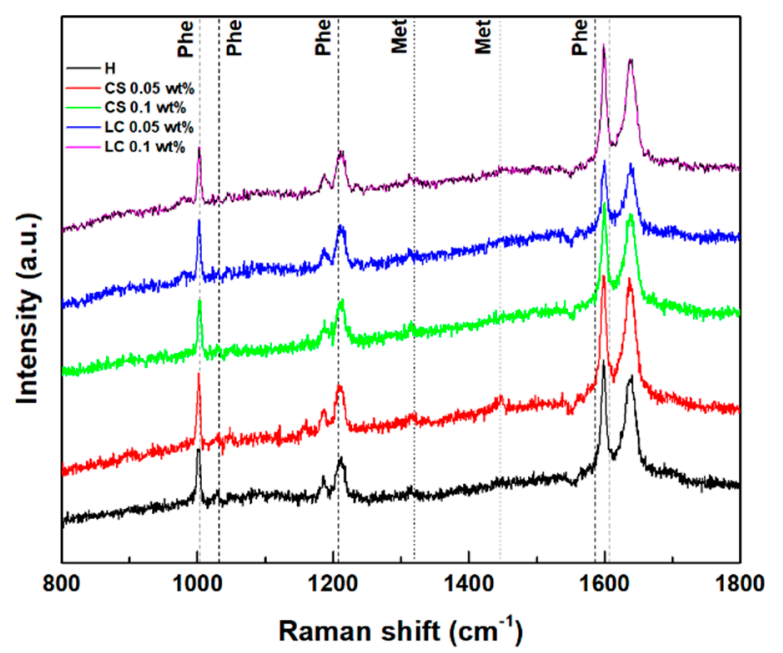

Figure 5. Raman scattering spectra of the hydrogel and magnetogels containing lipid-coated (LC) and citrate-stabilized (CS) nanoparticles. Vertical lines represent the reported Raman shifts of methionine (Met) and phenylalanine (Phe).

The Amide I region (1580-1700 $\mathrm{cm}^{-1}$ ) arises from the $\mathrm{C}=\mathrm{O}$ stretching vibration, which is sensitive to changes in backbone peptide conformation [61]. Deconvolution of the amide I band in its sub-bands is correlated with various secondary structure contributions (see Figure S5 in Supplementary Materials) [60-62]. A major contribution at $1637 \mathrm{~cm}^{-1}$ is common to all systems, which can be associated with a major $\beta$-sheet content $[61,62]$. The band at $1338 \mathrm{~cm}^{-1}$ also confirms the predominance of the $\beta$-sheet in all gels [61].

\subsection{Rheological Properties}

Large amplitude oscillatory shear strain sweeps (LAOSS) were carried out to assess the effect of the nanoparticles on the gels structure (Figure S6 in Supplementary Materials). Further, the effect of temperature at $37^{\circ} \mathrm{C}$ on the hydrogel shear elastic and loss moduli was also assessed (Figure S6), the decrease of which evidences the phase transition behavior observed in the microviscosity studies. The addition of nanoparticles reduced the elasticity of gels, as previously observed for other systems $[10,63]$. Interestingly, the lipid-coated nanoparticles induced a lower elasticity than the citrate-stabilized nanoparticles, which was similar to their influence on the gels' microviscosity. Further, the strain at which $\mathrm{G}^{\prime}$ crosses $G^{\prime \prime}$ increased in the presence of citrate-stabilized nanoparticles (at $0.05 \mathrm{wt} \%$ ). As a result, the profiles suggest that different structures were obtained for each nanoparticle content, compared to the bare gel. The lipid-coated nanoparticle-containing magnetogel displays the Payne effect characteristic of viscoelastic matrices reinforced by solid fillers, i.e., a local maximum of $G^{\prime \prime}$ concomitant with a significant decrease of $G^{\prime}$, which can be associated with the breakage and recovery of weak interaction bonds linking adjacent clusters, aggregation/disaggregation of nanoparticles, or molecular disentanglement [64].

\subsection{Electron Microscopy}

Figure 6 displays the STEM images of the citrate-stabilized and lipid-coated nanoparticles in solution and incorporated into the hydrogel matrix (magnetogels) prepared at $0.5 \mathrm{wt} \%$ of hydrogelator. The negative charge of the citrate molecules stabilizing the nanoparticles ensured there were well dispersed nanoparticles with an average size of $14.4 \pm 2.6 \mathrm{~nm}$ (see histograms in Figure S7 in Supplementary Materials), though some aggregates were observed (Figure 6A). Alternatively, the lipid-coated nanoparticles, with an 
average size of $8.9 \pm 2.1 \mathrm{~nm}$, displayed a tendency to form spherical aggregates, which can be associated with the dynamic membrane coating being prone to self-assembly (Figure 6B). The hydrogel network is also displayed for a matter of comparison with the magnetogels. The preparation conditions used here afforded a network comprised of thin and thick short fibers with a cross-section of $21.3 \pm 3.4 \mathrm{~nm}$ and $48.4 \pm 13.8 \mathrm{~nm}$, and with an average length of $1030 \pm 389 \mathrm{~nm}$.
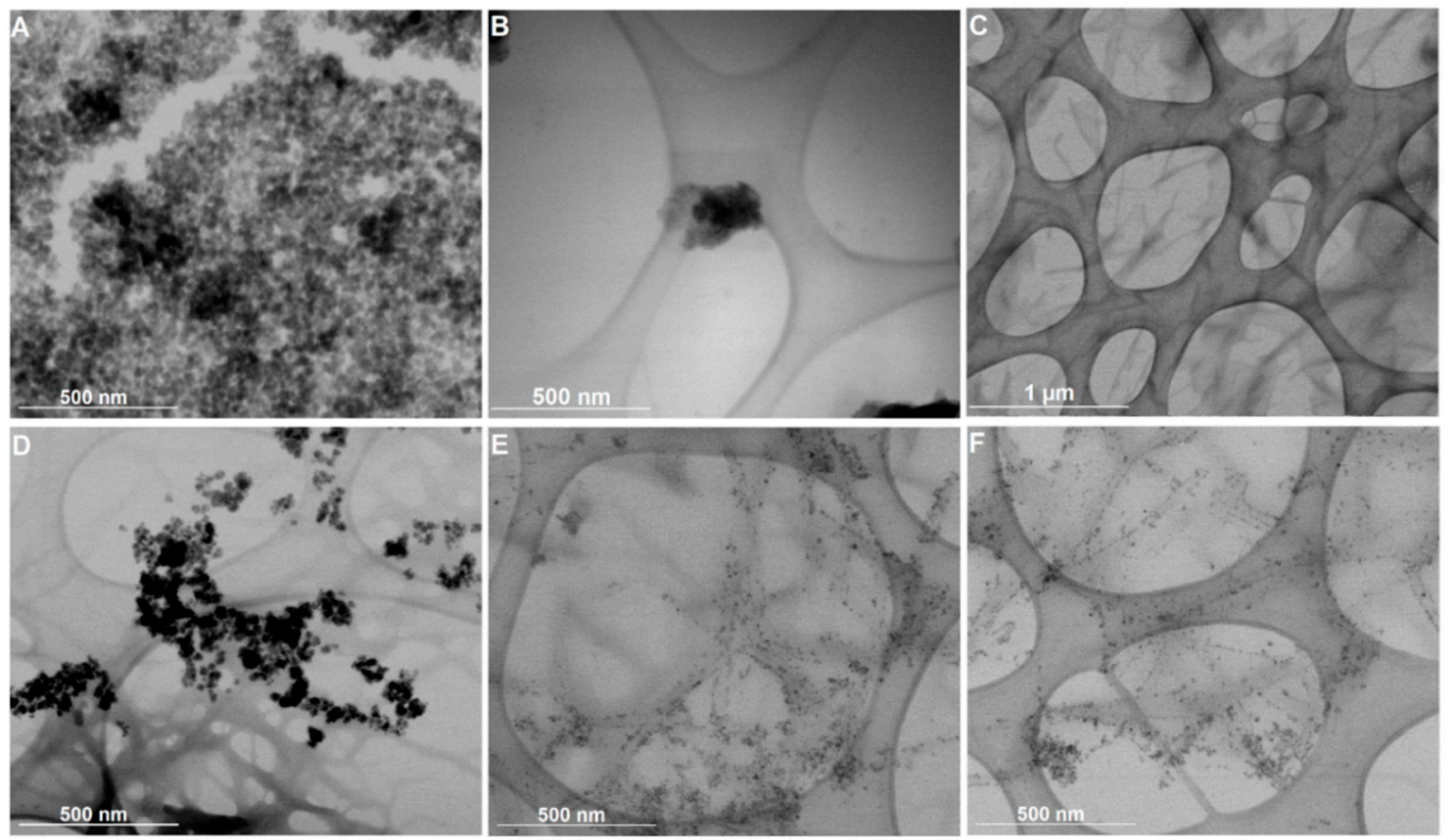

Figure 6. Scanning transmission electron microscopy (STEM) images of (A) citrate-stabilized nanoparticles, (B) lipidcoated nanoparticles, and (C) the hydrogel structure. Magnetogels containing (D) citrate-stabilized nanoparticles and (E,F) lipid-coated nanoparticles prepared at $0.5 \mathrm{wt} \%$ of hydrogelator, $1 \mathrm{wt} \%$ of GdL, and $0.05 \mathrm{wt} \%$ of nanoparticles.

Furthermore, different effects on the magnetogels microstructure were observed when using the two types of nanoparticles. The citrate-stabilized nanoparticles are randomly distributed within the hydrogel matrix as aggregates. This stems from the fact that the magnetogels based on $\mathrm{N}$-protected peptides lacked a cationic group in the hydrogelator structure electrostatically interacting with these negatively-charged stabilized nanoparticles and fixing them in the matrix. Nevertheless, the lipid-coated nanoparticles displayed an affinity towards the fibers surface, rendering them more adequate to avoid any potential leaking of nanoparticles. Furthermore, upon gelation, the lipid-coated nanoparticle aggregates become destabilized, as no aggregates were observed (see Figure 6E,F).

\subsection{Hyperthermia Studies}

The calorimetric approach was carried out to assess the magnetic nanoparticle hyperthermia effect in gels, while considering the medical threshold limit of $H_{0} f \leq 5 \times 10^{9} \mathrm{~A} \mathrm{~m}^{-1} \mathrm{~s}^{-1}[65,66]$, or $H_{0} f \leq 4.85 \times 10^{8} \mathrm{~A} \mathrm{~m}^{-1} \mathrm{~s}^{-1}$ [66], depending on the area exposed. The increase in temperature over time, when the nanoparticles are dispersed in aqueous solution and in gels while applying an alternating magnetic field, is displayed in Figure 7. 

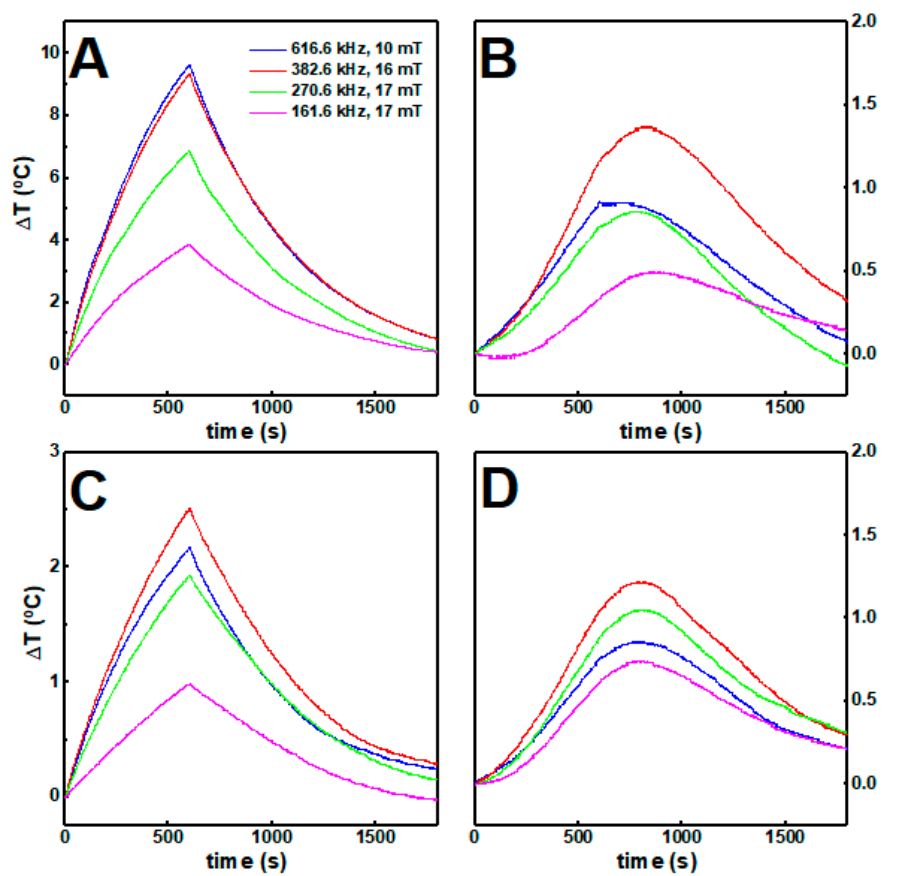

Figure 7. The temperature variation over time of (A) citrate-stabilized and (B) lipid-coated nanoparticles at $1 \mathrm{mg} / \mathrm{mL}(0.1 \mathrm{wt} \%)$ in water and in gels (C,D) respectively) under different magnetic field strengths and frequencies.

In water solution, the citrate-stabilized nanoparticles attained higher temperatures than the lipid-coated nanoparticles, which could be associated with the different stoichiometry in the spinel ferrites belonging to the two samples, with the different average sizes, and with the fact that the lipid-coated nanoparticles are more likely to aggregate in aqueous solution. In both cases, the increments in temperature decreased when the magnetic nanoparticles were incorporated in the gels, likely because some of the heat generated was employed for inducing local changes in the gel structure.

Furthermore, nanoparticles at high concentration within the gels underwent stronger magnetic dipolar interactions, which has a detrimental effect in the heat delivery capacity $[67,68]$. The heating efficiency was quantitatively evaluated through the intrinsic loss power (ILP) (see Table S6 in Supplementary Materials). While the ILP decreases for the citrate-stabilized nanoparticles around $80 \%$ when incorporated into the gels, for the lipidcoated nanoparticles no major changes were obtained. Accordingly, despite being less efficient than citrate-stabilized nanoparticles, the lipid-coated nanoparticles keep a similar heat delivery capacity when they are incorporated in the gels.

\subsection{Drug Release Assays}

\subsubsection{Incorporation of Doxorubicin}

Previously, supramolecular dehydropeptide-based hydrogels have shown promising results as drug delivery nanosystems [8]. Here, the nanoparticles concentration effect over doxorubicin incorporation is assessed. FRET (Förster Resonance Energy Transfer) process from the emissive moieties of the hydrogel aromatic moieties (acting as the energy donors) to doxorubicin (acting as the energy acceptor) allowed us to follow the encapsulation of doxorubicin owing to the overlap between the drug absorption band and hydrogel fluorescence emission (see Figure S8 in Supplementary Materials).

Fluorescence spectra of hydrogel and magnetogels at $0.025 \mathrm{wt} \%$ of citrate-stabilized and lipid-coated nanoparticles, with and without doxorubicin, are displayed in Figure 8. The absence of doxorubicin results in a strong fluorescence emission (Figure $8 \mathrm{~A}$ ) of the aggregates associated with the stacking of the aromatic rings $\left(\lambda_{\max } \sim 450 \mathrm{~nm}\right)$. 

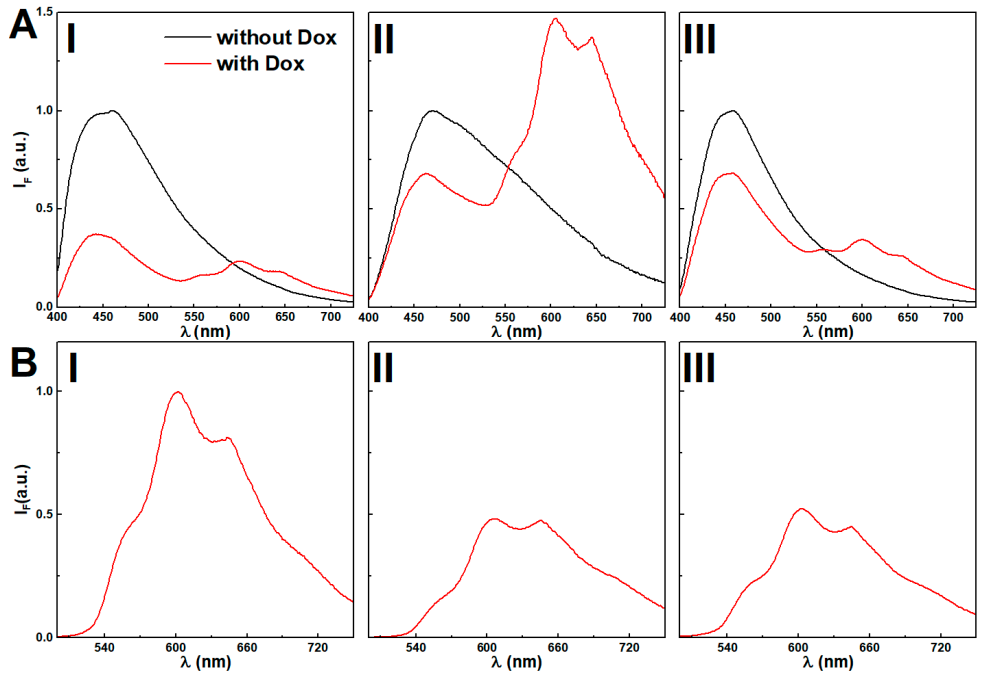

Figure 8. (A) Fluorescence emission spectra $\left(\lambda_{\mathrm{exc}}=375 \mathrm{~nm}\right)$ of hydrogel (I) and magnetogels containing citrate-stabilized (II) and lipid-coated (III) manganese ferrite nanoparticles $(0.025 \mathrm{wt} \%)$ incorporating the doxorubicin and comparison with the plain gels. (B) Fluorescence spectra $\left(\lambda_{\text {exc }}=480 \mathrm{~nm}\right)$ of directly-excited doxorubicin in gels, (I) and magnetogels con-taining citrate-stabilized (II) and lipid-coated (III) manganese ferrite nanoparticles $(0.025 \mathrm{wt} \%)$ incorporating the doxorubicin and comparison with the plain gels

The addition of citrate-stabilized nanoparticles induced a red-shift (Figure 8B), which was modulated by increasing the nanoparticle concentration (see Figure S9 in Supplementary Materials). The lipid-coated nanoparticles induced a thinning of the emission band shape, suggesting that the variety of microenvironments in the vicinity of the fibers was restrained.

Doxorubicin displays reverse solvatochromism, making the assignment of its location ambiguous [69]. Further, the assays with Nile Red demonstrated that a higher concentration of citrate-stabilized nanoparticles was observed to increase the polarity of the gel hydrophobic cavities. Thus, the decreasing fluorescence emission ratio of doxorubicin between the peaks at $\lambda=560 \mathrm{~nm}$ and $\lambda=600 \mathrm{~nm}$ upon addition of citrate-stabilized nanoparticles might be associated with an increasingly acidic region and its location in cavities, which are destabilized by the increasing concentration of nanoparticles (see Figure S10 in Supplementary Materials) [69]. Nonetheless, the possibility of aggregation is not excluded, considering that at $10 \mu \mathrm{M}$, a fraction of $47 \%$ is dimerized [70,71], and the obtained spectra in gels resemble the fluorescence emission spectrum of a doxorubicin concentrated solution $(0.1 \mathrm{mM})$ at $\mathrm{pH}=7$ (see Figure S11 in Supplementary Materials). The lipid-coated nanoparticles do not show the same effects, as the wavelength and emission ratio remain mostly unchanged, i.e., no major changes are induced in the doxorubicin location microenvironment. The fluorescence quenching by increasing nanoparticle concentration further suggests its proximity to the nanoparticles.

The hydrogelator fluorescence quantum yield at a nanoparticle concentration of $0.025 \mathrm{wt} \%$ was determined through Equation (7). The calculated FRET efficiencies $\left(\Phi_{\text {FRET }}\right)$, Förster radius $\left(R_{0}\right)$, and donor-acceptor distances $\left(R_{D A}\right)$ are presented in Table 1 . As reported in other systems $[8,63]$, the distances between the fiber aromatic groups and doxorubicin remain similar with or without nanoparticles, and suggests a host-guest type interaction $[8,63]$.

Here, similarly to the obtained results with Nile Red, the lipid-coated nanoparticles induced a lower anisotropy, while the fluorescence emission spectrum remains similar, suggesting the occurrence of lipid-fiber domains. Overall, the high anisotropy values suggest that doxorubicin has affinity towards the gel network fibers and the nanoparticles affect the arrangement of the fibers. 
Table 1. Förster Resonance Energy Transfer (FRET) efficiencies $\left(\Phi_{F R E T}\right)$, fluorescence quantum yields of the donor (hydrogel aggregates) $\left(\Phi_{D}^{0}\right)$, donor-acceptor distances $\left(R_{D A}\right)$ and steady-state fluorescence anisotropy $(r)$ values for gels with an incorporated drug. Anisotropy value of doxorubicin in glycerol (at $\left.25^{\circ} \mathrm{C}\right): \mathrm{r}=0.285$.

\begin{tabular}{ccccccc}
\hline System & Content (wt\%) & $\boldsymbol{\Phi}_{\text {FRET }}$ & $\boldsymbol{\Phi}_{\boldsymbol{D}}^{0}{ }^{\mathbf{a}}$ & $\begin{array}{c}\boldsymbol{R}_{0} \\
\mathbf{( n m )}\end{array}$ & $\begin{array}{c}\boldsymbol{R}_{\boldsymbol{D A}} \\
\mathbf{( n m )}\end{array}$ & $\boldsymbol{r}$ \\
\hline $\mathrm{H}$ & - & 0.66 & 0.012 & 2.1 & 1.9 & 0.17 \\
\hline \multirow{2}{*}{$\mathrm{CS}$} & 0.025 & 0.33 & 0.001 & 1.7 & 1.4 & 0.16 \\
& 0.05 & - & - & - & - & 0.15 \\
& 0.1 & - & - & - & - & 0.14 \\
\hline \multirow{2}{*}{$\mathrm{LC}$} & 0.025 & 0.33 & 0.010 & 2.3 & 2.0 & 0.13 \\
& 0.05 & - & - & - & - & 0.11 \\
& 0.1 & - & - & - & - & 0.11 \\
\hline
\end{tabular}

${ }^{\text {a }}$ Relative to L-tryptophan in aqueous buffer solution, $\mathrm{pH}=7.2\left(\Phi_{r}=0.14\right.$ at $\left.25^{\circ} \mathrm{C}\right)$ [39]. The error rate is about $10 \%$.

\subsubsection{Doxorubicin Release Assays}

In a previous drug release assay of hydrogels, a mixed behavior of gel erosion and drug diffusion was observed [8]. Doxorubicin release from the hydrogel and magnetogels to $\mathrm{pH}=7$ buffer (to keep $\mathrm{pH}$ conditions constant and neutralize gels) was assessed.

The release profiles of the hydrogel and both limiting conditions $(0.1 \mathrm{wt} \%$ of nanoparticles) of magnetogels are displayed in Figure 9A. Similarly to previous results for dehydropeptide hydrogels (without nanoparticles) and other magnetogels [62], an initial burst release occurs, followed by a slow release phase. Moreover, both systems display a low drug release profile that can be associated with the strong interactions established between the drug and gels components. Such profiles are useful for therapeutic applications, as the systems can be loaded with high amounts of chemotherapeutic drugs and ensure a prolonged and controlled release of a therapeutically relevant dose in the target site.
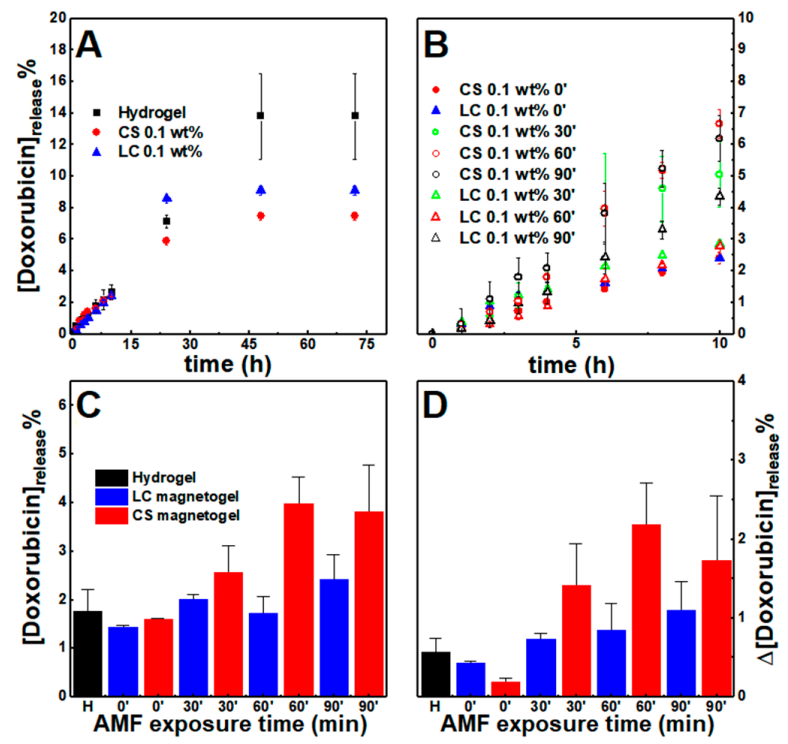

Figure 9. Cumulative doxorubicin percentage release profiles from the hydrogel and gels containing citrate-stabilized (CS) and lipid-coated (LC) nanoparticles (0.1 wt \%) (A) without an externally applied alternating magnetic field (AMF) and (B) comparison of the magnetogels release when an AMF is applied for 30,60, and $90 \mathrm{~min}$ between $\mathrm{t}=4 \mathrm{~h}$ and $\mathrm{t}=6 \mathrm{~h}$. (C) Cumulative doxorubicin released at $t=6 \mathrm{~h}$. (D) Variation of doxorubicin percentage released between $t=4 \mathrm{~h}$ and $t=6 \mathrm{~h}$, that is, for a period of $2 \mathrm{~h}$. 
Quantitative analysis of the cumulative drug release profiles without a magnetic field was carried out through the fitting of various mathematical models (see Tables S7 and S8 in Supplementary Materials) [72-75].

Overall, the obtained Gompertz model fitting suggests that both nanoparticles limited the maximum drug quantity released, while the Korsmeyer-Peppas mechanistic model $(0.45<\mathrm{n}<0.89)$ points to a mechanism that combines diffusion and erosion drug release (non-Fickian release) [72-74]. The latter contribution was also evidenced by the good fitting of the first-order model (associated with the diffusion of water-soluble drugs in porous matrices) and the Hixson-Crowell model (describes systems where changes in the surface area and diameter occur over time, but the initial geometrical shape is kept constant).

An alternating magnetic field (AMF) was applied for 30, 60, and $90 \mathrm{~min}, 2.98 \mathrm{mT}$ at $1000 \mathrm{kHz}$ after $4 \mathrm{~h}$, which resulted into an increase of cumulative doxorubicin release comparatively to the systems that were not subjected to the AMF (Figure 9B,C). To remove the contribution from preparation anomalies, the enhancement of drug release was further evidenced by comparing the amount of drug release in the period between $t=4 \mathrm{~h}$ and $\mathrm{t}=6 \mathrm{~h}$ (Figure 9D), that is, during two hours. The enhancement is larger in the citrate-stabilized nanoparticles than lipid-coated nanoparticles, which might be associated with the higher heating efficiency. However, the enhanced drug release displays a non-linear relation with the AMF exposure time. This can be associated with the nanoparticles being distributed as aggregates, as opposed to the lipid-coated nanoparticles that incrementally enhanced drug release, as the sample was exposed to longer periods. Further, gels subjected to the AMF for $30 \mathrm{~min}$ revealed a decrease of fluorescence anisotropy to 0.12 for the citrate-stabilized nanoparticles, while in the lipid-coated ones, it remained close to 0.15 , i.e., the citratestabilized nanoparticles heating induced an irreversible collapse of the gel network that led to an increased variance of drug released, while the other nanoparticles might have not affected the network structure.

As a result, the incremental drug release enhancement and retention of the lipid-coated nanoparticles developed here indicate that this supramolecular system architecture is a suitable approach for controlling drug release, since the heating-induced effect displayed improved reproducibility. However, lipid-coated nanoparticles with a higher heating efficiency and gels that can homogeneously include more nanoparticles are required in future developments to improve the percentage of drug released upon the AMF-trigger, as suggested from the higher triggered release from citrate-stabilized nanoparticle-containing gels.

\section{Conclusions}

Pursuing the effect of citrate and lipid-functionalized nanoparticles in the development of supramolecular magnetogels, the gelation of the hydrogel Cbz-L-Met-Z- $\Delta$ Phe-OH was systematically optimized by using kinetic models to prepare homogeneous magnetogels, while considering both the kinetics of gelation and sedimentation of nanoparticles. Lipidcoated nanoparticles formed lipid-fiber domains and increased the gel irreversible phase transition temperature. The heating efficiency of lipid-coated nanoparticles was improved (maximum heat and reproducibility) when they were incorporated in the gels, while a detrimental effect was obtained for citrate-stabilized nanoparticles. Further, the former did not produce major changes in doxorubicin encapsulation, while the latter increased the micropolarity of its location and induced aggregation. The magnetogels revealed similar doxorubicin release profiles and AMF-trigger was stronger in the citrate-stabilized nanoparticles, though the triggered release was more reproducible in the lipid-coated nanoparticle-containing gels.

Overall, the lipid-coated nanoparticles displayed promising results for future developments of supramolecular magnetogels aiming at the control of drug release. This was mainly associated with the improved nanoparticle distribution (along the hydrogel fibers), unaffected heating efficiency upon gelation, and reproducible triggered drug release. On the other hand, despite the higher heating efficiency of the negatively-charged citrate-stabilized nanoparticles in solution, they are prone to aggregation upon gelation, 
which is reflected in a decreased heating efficiency, and local inhomogeneous distribution consequently leading to less reproducibility in drug release.

Hereby, this work reveals that negatively-charged stabilized and lipid-coated nanoparticles affect the final gel architecture differently and, thus, also affect its properties and the encapsulation of drugs in different ways. Further, comparison of both systems points out that on-demand drug release in dehydropeptide-based supramolecular magnetogels can be optimized by developing nanoparticles that can adsorb onto hydrogel fibers, while providing domains that improve or do not affect drug encapsulation (lipid-coated nanoparticles). In particular, the unaffected drug encapsulation and reproducible release from the lipid-coated nanoparticle-containing gels, upon application of AMF, is anticipated to potentiate the supramolecular magnetic gels in drug delivery towards on-demand drug release.

Future developments will be focused on improving heating efficiency of the lipidcoated nanoparticles and the synergy between hyperthermia and triggered drug release, without inducing a major collapse of the hydrogel.

Supplementary Materials: The following are available online at https:/ /www.mdpi.com/2079-499 1/11/1/16/s1, Structure S1: Hydrogelator structure, Table S1: Final hydrogel pH, Figure S1: Knowles' aggregation model fitting to experimental aggregation profiles, Table S2: Calculated curve-fitting parameters of the turbidity profiles, Table S3: Calculated X-ray diffraction parameters, Table S4: Calculated curve-fitting parameters of the sedimentation profiles, Table S5: Obtained parameters from the SQUID hysteresis curves, Figure S2: dependence of $\frac{k_{\text {emp }}}{k_{\text {sed }}}$ on $s(t)$ and $f(t)$ for fixed $v$ and $a$ values, Figure S3: Image of the magnetogels containing citrate-stabilized and lipid-coated nanoparticles, Figure S4: Curve-fitting of the Raman scattering spectra in the wavelength range 960-1040 $\mathrm{cm}^{-1}$ and dependence of the Raman shift and full width at half maximum of the major phenyl ring vibration, Figure S5: Curve-fitting of the Raman scattering spectra of gels in the amide I region, Figure S6: Strain dependence of the shear elastic and loss moduli for gels, Figure S7: STEM images and histograms of gels, Table S6: Calculated magnetic hyperthermia parameters, Figure S8: Normalized fluorescence emission spectrum of the hydrogel and doxorubicin absorption spectrum, Figure S9: Normalized fluorescence emission spectra of hydrogel and magnetogels, Figure S10: Fluorescence emission spectra of doxorubicin in hydrogel and magnetogels at various nanoparticle concentration, Figure S11: Normalized fluorescence emission spectra of doxorubicin in $\mathrm{pH}=7$ buffer at various concentrations, Table S7: Coefficients of determination for doxorubicin release profiles, Table S8: Release coefficients of the models fitted to the experimental release profiles.

Author Contributions: Conceptualization, S.R.S.V., P.M.T.F., and E.M.S.C.; methodology, S.R.S.V., L.H., C.M., P.J.G.C., J.A.M., V.S., M.A.C.-D., P.M.T.F., and E.M.S.C.; formal analysis, S.R.S.V., J.F.G.S., L.H., C.M., P.J.G.C., J.A.M., M.T.-A., V.S., M.A.C.-D., P.M.T.F., and E.M.S.C.; investigation, S.R.S.V., J.F.G.S., L.H., C.M., M.T.-A., V.S., M.A.C.-D., P.M.T.F., and E.M.S.C.; writing-original draft preparation, S.R.S.V., P.M.T.F., and E.M.S.C.; writing-review and editing, M.A.C.-D. and E.M.S.C.; supervision, M.A.C.-D., P.M.T.F., and E.M.S.C.; project administration, P.J.G.C. and P.M.T.F. All authors have read and agreed to the published version of the manuscript.

Funding: This work was supported by the Portuguese Foundation for Science and Technology (FCT) in the framework of the Strategic Funding of CF-UM-UP (UIDB/04650/2020) and CQUM (UIDB/00686/2020). FCT, FEDER, PORTUGAL2020 and COMPETE2020 are also acknowledged for funding under research projects PTDC/QUI-QFI/28020/2017 (POCI-01-0145-FEDER-028020), PTDC/QUI-QOR/29015/2017 (POCI-01-0145-FEDER-029015), UIDB/05256/2020 and UIDP/05256/2020. L.H. acknowledges the funding from the "FCT Researcher" Programme through grant IF/00606/2014. S. R. S. Veloso acknowledges FCT for a PhD grant (SFRH/BD/144017/2019). Support from MAP-Fis Doctoral Programme is also acknowledged. Ministerio de Economía y Competitividad (MINECO, Spain) under the Grant (CTM2017-84050-R), Xunta de Galicia/FEDER (IN607A 2018/5 and Centro Singular de Investigación de Galicia accreditation 2019-2022, ED431G 2019/06), 0712_ACUINANO_1_E,0624_2IQBIONEURO_6_E and NANOCULTURE cofounded by FEDER through the program Interreg V-A España-Portugal (POCTEP), NANOCULTURE (ERDF: 1.102.531) Interreg Atlantic Area, the European Union (European Regional Development Fund-ERDF).

Conflicts of Interest: The authors declare no conflict of interest. 


\section{References}

1. Ma, M.; Kuang, Y.; Gao, Y.; Zhang, Y.; Gao, P.; Xu, B. Aromatic-aromatic interactions induce the self-assembly of pentapeptidic derivatives in water to form nanofibers and supramolecular hydrogels. J. Am. Chem. Soc. 2010, 132, 2719-2728. [CrossRef] [PubMed]

2. Sutton, S.; Campbell, N.L.; Cooper, A.I.; Kirkland, M.; Frith, W.J.; Adams, D.J. Controlled release from modified amino acid hydrogels governed by molecular size or network dynamics. Langmuir 2009, 25, 10285-10291. [CrossRef] [PubMed]

3. Bhattacharya, S.; Samanta, S. Soft-nanocomposites of nanoparticles and nanocarbons with supramolecular and polymer gels and their applications. Chem. Rev. 2016, 116, 11967-12028. [CrossRef] [PubMed]

4. Jian, H.; Wand, M.; Dong, Q.; Li, J.; Wang, A.; Li, X.; Ren, P.; Bai, S. Dipeptide self-assembled hydrogels with tunable mechanical properties and degradability for 3D bioprinting. ACS Appl. Mater. Interfaces 2019, 11, 46419-46426. [CrossRef] [PubMed]

5. Fichman, G.; Gazit, E. Self-assembly of short peptides to form hydrogels: Design of building blocks, physical properties and technological applications. Acta Biomater. 2014, 10, 1671-1682. [CrossRef] [PubMed]

6. Yuan, D.; Bing, X. Heterotypic supramolecular hydrogels. J. Mater. Chem. B 2016, 4, 5638-5649. [CrossRef]

7. Draper, E.R.; Adams, D.J. Low-molecular-weight gels: The state of the art. Chem 2017, 2, 716-731. [CrossRef]

8. Veloso, S.R.S.; Magalhães, C.A.B.; Rodrigues, A.R.O.; Vilaça, H.; Queiroz, M.J.R.P.; Martins, J.A.; Coutinho, P.J.G.; Ferreira, P.M.T.; Castanheira, E.M.S. Novel dehydropeptide-based magnetogels containing manganese ferrite nanoparticles as antitumor drug nanocarriers. Phys. Chem. Chem. Phys. 2019, 21, 10377-10390. [CrossRef]

9. Veloso, S.R.S.; Ferreira, P.M.T.; Martins, J.A.; Coutinho, P.J.G.; Castanheira, E.M.S. Core-shell magnetic-plasmonic nanoparticles enclosed in a biocompatible dehydropeptide-based hydrogel containing lysine. In Proceedings of the Fourth International Conference on Applications of Optics and Photonics, Lisbon, Portugal, 3 October 2019.

10. Carvalho, A.; Gallo, J.; Pereira, D.M.; Valentão, P.; Andrade, P.B.; Hilliou, L.; Ferreira, P.M.T.; Bañobre-Lopez, M.; Martins, J.A. Magnetic dehydrodipeptide-based self-assembled hydrogels for theragnostic applications. Nanomaterials 2019, 9, 541. [CrossRef]

11. Ali, S.; Yosipovitch, G. Skin pH: From basic science to basic skin care. Acta Derm. Venereol. 2013, 93, 261-267. [CrossRef]

12. Bonhome-Espinosa, A.; Campos, F.; Rodriguez, I.; Carriel, V.; Marins, J.; Zubarev, A.; Duran, J.; Lopez-Lopez, M. Effect of particle concentration on the microstructural and macromechanical properties of biocompatible magnetic hydrogels. Soft Matter 2017, 13, 2928-2941. [CrossRef] [PubMed]

13. Antman-Passig, M.; Shefi, O. Remote magnetic orientation of 3D collagen hydrogels for directed neuronal regeneration. Nano Lett. 2016, 16, 2567-2573. [CrossRef] [PubMed]

14. Kim, H.; Jo, A.; Baek, A.; Lim, D.; Park, S.; Cho, S.; Chung, J.; Yoon, J. Synergistically enhanced selective intracellular uptake of anticancer drug carrier comprising folic acid-conjugated hydrogels containing magnetite nanoparticles. Sci. Rep. 2017, 7, 1-10. [CrossRef] [PubMed]

15. Testa-Anta, M.; Liébana-Viñas, S.; Rivas-Murias, B.; Rodríguez-González, B.; Farle, M.; Salgueiriño, V. Shaping iron oxide nanoparticles for magnetic separation applications. Nanoscale 2018, 10, 20462-20467. [CrossRef]

16. Munaweera, I.; Shi, Y.; Koneru, B.; Saez, R.; Aliev, A.; Di Pasqua, A.; Balkus, K. Chemoradiotherapeutic magnetic nanoparticles for targeted treatment of nonsmall cell lung cancer. Mol. Pharm. 2015, 12, 3588-3596. [CrossRef]

17. Hervault, A.; Thanh, N. Magnetic nanoparticle-based therapeutic agents for thermo-chemotherapy treatment of cancer. Nanoscale 2014, 6, 11553-11573. [CrossRef]

18. Akbarzadeh, A.; Samiei, M.; Davaran, S. Magnetic nanoparticles: Preparation, physical properties, and applications in biomedicine. Nanoscale Res. Lett. 2012, 7, 144. [CrossRef]

19. Veloso, S.R.S.; Ferreira, P.M.T.; Martins, J.A.; Coutinho, P.J.G.; Castanheira, E.M.S. Magnetogels: Prospects and main challenges in biomedical applications. Pharmaceutics 2018, 10, 145. [CrossRef]

20. Zhang, Z.Q.; Song, S.C. Thermosensitive/superparamagnetic iron oxide nanoparticle-loaded nanocapsule hydrogels for multiple cancer hyperthermia. Biomaterials 2016, 106, 13-23. [CrossRef]

21. Ramos-Docampo, M.; Fernández-Medina, M.; Taipaleenmäki, E.; Hovorka, O.; Salgueiriño, V.; Stadler, B. Microswimmers with heat delivery capacity for 3D cell spheroid penetration. Acs Nano 2019, 13, 12192-12205. [CrossRef]

22. Bhardwaj, A.; Parekh, K.; Jain, N. In vitro hyperthermic effect of magnetic fluid on cervical and breast cancer cells. Sci. Rep. 2020, 10, 15249. [CrossRef] [PubMed]

23. Guisasola, E.; Asín, L.; Beola, L.; de la Fuente, J.; Baeza, A.; Vallet-Regí, M. Beyond Traditional Hyperthermia: In Vivo Cancer Treatment with Magnetic-Responsive Mesoporous Silica Nanocarriers. Acs Appl. Mater. Interfaces 2018, 10, 12518-12525. [CrossRef] [PubMed]

24. Amiri, M.; Gholami, T.; Amiri, O.; Pardakhti, A.; Ahmadi, M.; Akbari, A.; Amanatfard, A.; Salavati-Niasari, M. The magnetic inorganic-organic nanocomposite based on $\mathrm{ZnFe}_{2} \mathrm{O}_{4}$-Imatinib-liposome for biomedical applications, in vivo and in vitro study. $J$. Alloy. Compd. 2020, 849, 156604. [CrossRef]

25. Colombo, M.; Carregal-Romero, S.; Casula, M.F.; Gutiérrez, L.; Morales, M.P.; Böhm, I.B.; Heverhagen, J.T.; Prosperi, D.; Parak, W.J. Biological applications of magnetic nanoparticles. Chem. Soc. Rev. 2012, 41, 4306-4334. [CrossRef]

26. Gila-Vilchez, C.; Mañas-Torres, M.; Contreras-Montoya, R.; Alaminos, M.; Duran, J.; de Cienfuegos, L.; Lopez-Lopez, M. Anisotropic magnetic hydrogels: Design, structure and mechanical properties. Philos. Trans. R. Soc. A 2019, $377,20180217$. [CrossRef] 
27. Yang, Z.; Gu, H.; Du, J.; Gao, J.; Zhang, B.; Zhang, X.; Xu, B. Self-assembled hybrid nanofibers confer a magnetorheological supramolecular hydrogel. Tetrahedron 2007, 63, 7349-7357. [CrossRef]

28. Nawara, K.; Romiszewski, J.; Kijewska, K.; Szczytko, J.; Twardowski, A.; Mazur, M.; Krysinski, P. Adsorption of doxorubicin onto citrate-stabilized magnetic nanoparticles. J. Phys. Chem. C 2012, 116, 5598-5609. [CrossRef]

29. Hui, C.; Shen, C.; Yang, T.; Bao, L.; Tian, J.; Ding, H.; Li, C.; Gao, H.J. Large-scale $\mathrm{Fe}_{3} \mathrm{O}_{4}$ nanoparticles soluble in water synthesized by a facile method. J. Phys. Chem. C 2008, 112, 11336-11339. [CrossRef]

30. Pagano, R.; López Medus, M.; Gómez, G.; Couto, P.; Labanda, M.; Landolfo, L.; D’Alessio, C.; Caramelo, J. Protein fibrillation lag times during kinetic inhibition. Biophys. J. 2014, 107, 711-720. [CrossRef]

31. Wang, S.; Chen, Y.; Chen, P.; Liu, K. A kinetic study on the aggregation behavior of $\beta$-amyloid peptides in different initial solvent environments. Biochem. Eng. J. 2006, 29, 129-138. [CrossRef]

32. Morris, A.; Watzky, M.; Finke, R. Protein aggregation kinetics, mechanism, and curve-fitting: A review of the literature. Biochim. Biophys. Actaproteins Proteom. 2009, 1794, 375-397. [CrossRef] [PubMed]

33. Kamihira, M.; Naito, A.; Tuzi, S.; Saitô, H.; Nosaka, A. Conformational transitions and fibrillation mechanism of human calcitonin as studied by high-resolution solid-state 13C NMR. Protein Sci. 2000, 9, 867-877. [CrossRef] [PubMed]

34. Knowles, T.; Waudby, C.; Devlin, G.; Cohen, S.; Aguzzi, A.; Vendruscolo, M.; Terentjev, E.; Welland, M.; Dobson, C. An analytical solution to the kinetics of breakable filament assembly. Science 2009, 326, 1533-1537. [CrossRef] [PubMed]

35. Cohen, S.; Linse, S.; Luheshi, L.; Hellstrand, E.; White, D.; Rajah, L.; Otzen, D.; Vendruscolo, M.; Dobson, C.; Knowles, T. Proliferation of amyloid- $\beta 42$ aggregates occurs through a secondary nucleation mechanism. Proc. Natl. Acad. Sci. USA 2013, 110, 9758-9763. [CrossRef]

36. Crosby, G.A.; Demas, J.N. The measurement of photoluminescence quantum yields-Review. J. Phys. Chem. 1971, 75, 991-1024. [CrossRef]

37. Fery-Forgues, S.; Lavabre, D. Are fluorescence quantum yields so tricky to measure? A demonstration using familiar stationery products. J. Chem. Educ. 1999, 76, 1260-1264. [CrossRef]

38. Kirby, E.P.; Steiner, R.F. Influence of solvent and temperature upon the fluorescence of indole derivatives. J. Phys. Chem. 1970, 74, 4480-4490. [CrossRef]

39. Valeur, B. Molecular Fluorescence-Principles and Applications, 2nd ed.; Wiley-VCH: Weinheim, Germany, 2001.

40. Cardoso, A.; Alvarez, A.; Cattoz, B.; Griffiths, P.; King, S.; Frith, W.; Adams, D. The influence of the kinetics of self-assembly on the properties of dipeptide hydrogels. Faraday Discuss. 2013, 166, 101-116. [CrossRef]

41. Greenspan, P.; Fowler, S. Spectrofluorometric studies of the lipid probe Nile Red. J. Lipid Res. 1985, 26, 781-789.

42. Ira; Krishnamoorthy, G. Probing the link between proton transport and water content in lipid membranes. J. Phys. Chem. B 2001, 105, 1484-1488. [CrossRef]

43. Coutinho, P.J.G.; Castanheira, E.M.S.; Rei, M.C.; Oliveira, M.E.C.D.R. Nile Red and DCM fluorescence anisotropy studies in $\mathrm{C}_{12} \mathrm{E}_{7}$ /DPPC Mixed Systems. J. Phys. Chem. B 2002, 106, 12841-12846. [CrossRef]

44. Hungerford, G.; Castanheira, E.M.S.; Real Oliveira, M.E.C.D.; Miguel, M.G.; Burrows, H. Monitoring ternary systems of $\mathrm{C}_{12} \mathrm{E}_{5}$ /water/tetradecane via the fluorescence of solvato-chromic probes. J. Phys. Chem. B 2002, 106, 4061-4069. [CrossRef]

45. Rodrigues, A.R.O.; Matos, J.O.G.; Nova Dias, A.M.; Almeida, B.G.; Pires, A.; Pereira, A.M.; Araújo, J.P.; Queiroz, M.J.R.P.; Castanheira, E.M.S.; Coutinho, P.J.G. Development of multifunctional liposomes containing magnetic/plasmonic $\mathrm{MnFe}_{2} \mathrm{O}_{4} / \mathrm{Au}$ core/shell nanoparticles. Pharmaceutics 2019, 11, 10. [CrossRef] [PubMed]

46. Cardoso, B.D.; Rodrigues, A.R.O.; Almeida, B.G.; Amorim, C.O.; Amaral, V.S.; Castanheira, E.M.S.; Coutinho, P.J.G. Stealth magnetoliposomes based on calcium-substituted magnesium ferrite nanoparticles for curcumin transport and release. Int. J. Mol. Sci. 2020, 21, 3641. [CrossRef] [PubMed]

47. Pitschke, W.; Hermann, H.; Mattern, N. The influence of surface roughness on diffracted X-ray intensities in Bragg-Brentano geometry and its effect on the structure determination by means of Rietveld analysis. Powder Diffr. 1993, 8, 74-83. [CrossRef]

48. Lisboa, F.S.; Gardolinski, J.E.F.C.; Cordeiro, C.S.; Wypych, F. Layered metal laurates as active catalysts in the methyl/ethyl esterification reactions of lauric acid. J. Braz. Chem. Soc. 2012, 23, 46. [CrossRef]

49. Rodrigues, A.R.O.; Ramos, J.M.F.; Gomes, I.T.; Almeida, B.G.; Araújo, J.P.; Queiroz, M.J.R.P.; Coutinho, P.J.G.; Castanheira, E.M.S Magnetoliposomes based on manganese ferrite nanoparticles as nanocarriers for antitumor drugs. RSC Adv. 2016, 6, 17302-17313. [CrossRef]

50. Foderà, V.; Donald, A. Tracking the heterogeneous distribution of amyloid spherulites and their population balance with free fibrils. Eur. Phys. J. E 2010, 33, 273-282. [CrossRef]

51. Hsiung, C.; Lien, H.; Galliano, A.; Yeh, C.; Shih, Y. Effects of water chemistry on the destabilization and sedimentation of commercial $\mathrm{TiO}_{2}$ nanoparticles: Role of double-layer compression and charge neutralization. Chemosphere 2016, 151, 145-151. [CrossRef]

52. Phenrat, T.; Saleh, N.; Sirk, K.; Tilton, R.; Lowry, G. Aggregation and sedimentation of aqueous nanoscale zerovalent iron dispersions. Environ. Sci. Technol. 2007, 41, 284-290. [CrossRef]

53. Obaidat, I.; Issa, B.; Haik, Y. Magnetic properties of magnetic nanoparticles for efficient hyperthermia. Nanomaterials 2015, 5, 63-89. [CrossRef] [PubMed]

54. Smit, J. Magnetic Properties of Materials; McGraw Hill: New York, NY, USA, 1971. 
55. Swain, J.; Mishra, A. Nile red fluorescence for quantitative monitoring of micropolarity and microviscosity of pluronic F127 in aqueous media. Photochem. Photobiol. Sci. 2016, 15, 1400-1407. [CrossRef] [PubMed]

56. Swain, J.; Mishra, J.; Ghosh, G.; Mishra, A. Quantification of micropolarity and microviscosity of aggregation and salt-induced gelation of sodium deoxycholate (NaDC) using Nile red fluorescence. Photochem. Photobiol. Sci. 2019, 18, 2773-2781. [CrossRef] [PubMed]

57. Marchi, M.; Bilmes, S.; Negri, R. Microviscosity in the cavities of titania gels studied by steady-state fluorescence anisotropy. Langmuir 1997, 13, 3665-3673. [CrossRef]

58. Hernández, B.; Pflüger, F.; Kruglik, S.; Ghomi, M. Characteristic Raman lines of phenylalanine analyzed by a multiconformational approach. J. Raman Spectrosc. 2013, 44, 827-833. [CrossRef]

59. Noothalapati, H.; Iwasaki, K.; Yoshimoto, C.; Yoshikiyo, K.; Nishikawa, T.; Ando, M.; Hamaguchi, H.; Yamamoto, T. Imaging phospholipid conformational disorder and packing in giant multilamellar liposome by confocal Raman microspectroscopy. Spectrochim. Acta A Mol. Biomol. Spectrosc. 2017, 187, 186-190. [CrossRef]

60. Kurouski, D.; Van Duyne, R.; Lednev, I. Exploring the structure and formation mechanism of amyloid fibrils by Raman spectroscopy: A review. Analyst 2015, 140, 4967-4980. [CrossRef]

61. Kuhar, N.; Sil, S.; Verma, T.; Umapathy, S. Challenges in application of Raman spectroscopy to biology and materials. RSC Adv. 2018, 8, 25888-25908. [CrossRef]

62. Maiti, N.; Apetri, M.; Zagorski, M.; Carey, P.; Anderson, V. Raman spectroscopic characterization of secondary structure in natively unfolded proteins: $\alpha$-Synuclein. J. Am. Chem. Soc. 2004, 126, 2399-2408. [CrossRef]

63. Veloso, S.R.S.; Martins, J.A.; Hilliou, L.; Amorim, C.O.; Amaral, V.S.; Almeida, B.G.; Jervis, P.J.; Moreira, R.; Pereira, D.M.; Coutinho, P.J.G.; et al. Dehydropeptide-based plasmonic magnetogels: A supramolecular composite nanosystem for multimodal cancer therapy. J. Mater. Chem. B 2020, 8, 45-64. [CrossRef]

64. Fan, X.; Xu, H.; Zhang, Q.; Xiao, D.; Song, Y.; Zheng, Q. Insight into the weak strain overshoot of carbon black filled natural rubber. Polymer 2019, 167, 109-117. [CrossRef]

65. Mehdaoui, B.; Meffre, A.; Carrey, J.; Lachaize, S.; Lacroix, L.; Gougeon, M.; Chaudret, B.; Respaud, M. Optimal size of nanoparticles for magnetic hyperthermia: A combined theoretical and experimental study. Adv. Funct. Mater. 2011, 21, 4573-4581. [CrossRef]

66. Umut, E.; Coşkun, M.; Pineider, F.; Berti, D.; Güngüneş, H. Nickel ferrite nanoparticles for simultaneous use in magnetic resonance imaging and magnetic fluid hyperthermia. J. Colloid Interface Sci. 2019, 550, 199-209. [CrossRef] [PubMed]

67. Mehdaoui, B.; Tan, R.; Meffre, A.; Carrey, J.; Lachaize, S.; Chaudret, B.; Respaud, M. Increase of magnetic hyperthermia efficiency due to dipolar interactions in low-anisotropy magnetic nanoparticles: Theoretical and experimental results. Phys. Rev. B 2013, 87, 174419. [CrossRef]

68. Abenojar, E.; Wickramasinghe, S.; Bas-Concepcion, J.; Samia, A. Structural effects on the magnetic hyperthermia properties of iron oxide nanoparticles. Prog. Nat. Sci-Mater. 2016, 26, 440-448. [CrossRef]

69. Karukstis, K.; Thompson, E.; Whiles, J.; Rosenfeld, R. Deciphering the fluorescence signature of daunomycin and doxorubicin Biophys. Chem. 1998, 73, 249-263. [CrossRef]

70. Tasca, E.; Alba, J.; Galantini, L.; D’Abramo, M.; Giuliani, A.; Amadei, A.; Palazzo, G.; Giustini, M. The self-association equilibria of doxorubicin at high concentration and ionic strength characterized by fluorescence spectroscopy and molecular dynamics simulations. Colloids Surf. A 2019, 577, 517-522. [CrossRef]

71. Changenet-Barret, P.; Gustavsson, T.; Markovitsi, D.; Manet, I.; Monti, S. Unravelling molecular mechanisms in the fluorescence spectra of doxorubicin in aqueous solution by femtosecond fluorescence spectroscopy. Phys. Chem. Chem. Phys. 2013, 15, 2937. [CrossRef]

72. Dash, S.; Murthy, P.N.; Nath, L.; Chowdhury, P. Kinetic modelling on drug release from controlled drug delivery systems. Acta Pol. Pharm. 2010, 67, 217-223.

73. Ghitman, J.; Stan, R.; Ghebaur, A.; Cecoltan, S.; Vasile, E.; Iovu, H. Novel PEG-modified hybrid PLGA-vegetable oils nanostructured carriers for improving performances of indomethacin delivery. Polymers 2018, 10, 579. [CrossRef]

74. Kini, S.; Bahadur, D.; Panda, D. Mechanism of anti-cancer activity of benomyl loaded nanoparticles in multidrug resistant cancer cells. J. Biomed. Nanotechnol. 2015, 11, 877-889. [CrossRef] [PubMed]

75. Joy, M.; Iyengar, S.; Chakraborty, J.; Ghosh, S. Layered double hydroxide using hydrothermal treatment: Morphology evolution, intercalation and release kinetics of diclofenac sodium. Front. Mater. Sci. 2017, 11, 395-408. [CrossRef] 